\title{
Regular Bulk Solutions in Brane-Worlds with Inhomogeneous Dust and Generalized Dark Radiation
}

\author{
A. Herrera-Aguilar, ${ }^{1,2}$ A. M. Kuerten, ${ }^{3,4}$ and Roldão da Rocha ${ }^{5,6}$ \\ ${ }^{1}$ Departamento de Física, Universidad Autónoma Metropolitana Iztapalapa, San Rafael Atlixco 186, 09340 México, DF, Mexico \\ ${ }^{2}$ Instituto de Física y Matemáticas, Universidad Michoacana de San Nicolás de Hidalgo, Ciudad Universitaria, \\ 58040 Morelia, MICH, Mexico \\ ${ }^{3} \mathrm{CCNH}$, Universidade Federal do ABC, 09210-580 Santo André, SP, Brazil \\ ${ }^{4}$ Instituto de Ciencias Físicas, Universidad Nacional Autónoma de México, MEX, 62210 Cuernavaca, MOR, Mexico \\ ${ }^{5}$ CMCC, Universidade Federal do ABC, 09210-580 Santo André, SP, Brazil \\ ${ }^{6}$ International School for Advanced Studies (SISSA), Via Bonomea 265, 34136 Trieste, Italy
}

Correspondence should be addressed to Roldão da Rocha; roldao.rocha@ufabc.edu.br

Received 13 September 2014; Revised 18 December 2014; Accepted 18 December 2014

Academic Editor: Elias C. Vagenas

Copyright (C) 2015 A. Herrera-Aguilar et al. This is an open access article distributed under the Creative Commons Attribution License, which permits unrestricted use, distribution, and reproduction in any medium, provided the original work is properly cited. The publication of this article was funded by SCOAP ${ }^{3}$.

From the dynamics of a brane-world with matter fields present in the bulk, the bulk metric and the black string solution near the brane are generalized, when both the dynamics of inhomogeneous dust/generalized dark radiation on the brane-world and inhomogeneous dark radiation in the bulk as well are considered as exact dynamical collapse solutions. Based on the analysis on the inhomogeneous static exterior of a collapsing sphere of homogeneous dark radiation on the brane, the associated black string warped horizon is studied, as well as the 5D bulk metric near the brane. Moreover, the black string and the bulk are shown to be more regular upon time evolution, for suitable values for the dark radiation parameter in the model, by analyzing the soft physical singularities.

\section{Introduction}

Brane-world models with a single extra dimension $[1,2]$ are decidedly a straightforward 5D phenomenological realization of Hořava-Witten supergravity solutions [3], when the moduli effects from compact extra dimensions can be ignored (for a review, see, e.g., [4]). The Hořava-Witten solution [3] can be thought of as effectively 5 -dimensional, with an extra dimension that can be large relative to the fundamental scale. They provide the basis for the Randall-Sundrum (RS) braneworld models [2] that have mirror symmetry and a brane with tension as well, which counter balances the influence of the negative bulk cosmological constant on the brane, encompassing furthermore self-gravity of the branes [4]. In RS brane-world scenarios, our universe is embedded in a $5 \mathrm{D}$ bulk of type $\mathrm{AdS}_{5}$ [2]. The formalism to be used hereon employs a general metric for the brane-world, instead of the Minkowski metric in the standard RS model [2].
Brane-world black holes were comprehensively studied in Randall-Sundrum like brane-world cosmologies [5-7], where the dynamical equations on the brane are different from the general relativity ones. In fact, the brane-world framework presents terms that handle both the effects of the free gravitational field in the bulk and of the brane embedding in the bulk as well. The local energy-momentum corrections are usually dismissed, except at the high energy regime. The imprint on the brane of the nonlocal gravitational field in the bulk provides a splitting into anisotropic stress, flux, and nonlocal energy density. The nonlocal energy density determines the tidal acceleration in the off-brane direction and can oppose singularity formation [4]. Unlike the nonlocal energy density and flux, the nonlocal anisotropic stress is not determined by an evolution equation on the brane, reflecting the fact that brane observers cannot in general make predictions from initial data. In particular, isotropy of the cosmic microwave background may no longer guarantee a Friedmann geometry. 
Adiabatic density perturbations are coupled to perturbations in the nonlocal bulk field, and in general the system is not closed on the brane [7].

Bulk effects in the cosmological dynamics of brane-world scenarios have been studied [8], also in the context of thick brane-worlds [9-13], brane-worlds with variable tension [1417], and Kalb-Ramond fields [18], as well as in the context of more general brane-worlds and bulks [19-23]. Moreover, consequences of the gravitational collapse were proposed in the context of brane-world scenarios in, for example, [24-28]. In addition, dark matter was investigated already in [29] as a bulk effect on the brane.

Black strings can be thought as being extended objects endowed with an event horizon, in low energy string theory [30]. The bulk metric near the brane and the black string warped horizon along the extra dimension are here reviewed, based on previous developments [4, 31]. Originally, a Schwarzschild black hole on the brane-world was shown to be a black string in a higher dimensional spacetime, what leads to the usual astrophysical properties of black holes to be recovered in this scenario [31]. In this prototypical and prominent context, the Kretschmann curvature invariants diverge when the black string event horizon is approached along the axis of the black string. Several generalizations provide attempts to preclude singularities both in the bulk and on the brane-world. When variable brane tension scenarios are taken into account, the brane tension can gauge the Kretschmann scalars involved. For instance, regular bulk solutions and black strings were obtained in FriedmannRobertson-Walker brane-worlds under the Eötvös law [32], where the singularities related to the McVittie metric can be partially controlled as the cosmological time elapses. Indeed, for this metric the 5D soft physical singularities in the bulk are alleviated as time elapses, providing a regular 5D bulk solution, as the 5D Kretschmann invariants do not diverge. When other metrics are taken into account, for instance, the Casadio-Fabbri-Mazzacurati metric one [33], black strings can be still emulated [34]; however the related singularities in the bulk remain, regardless. In order to accomplish it, effective approaches are usually employed, where the black string is made to evolve from the brane-world [4, 31, 35].

Brane solutions of static black hole exteriors with 5D corrections to the Schwarzschild metric have been found, for instance, in [36-38] and furthermore in the context where the bulk singularities can be removed [39]. The (Schwarzchild) black string is unstable near the $\mathrm{AdS}_{5}$ horizon, defining the so-called Gregory-Laflamme instability [40, 41]. This scenario might be drastically altered by the inhomogeneous dust and the dark radiation. In order to accomplish this effect, we use a procedure to calculate both the metric near the brane and the 5D black string horizon [15], uniquely from a brane-world black hole metric and the associated Weyl tensor. Based on the knowledge of both the SasakiShiromizu-Maeda effective field equations on the brane and upon the 5D Einstein and Bianchi equations [4, 7, 35, 42, 43], both the bulk metric near the brane and in particular the black string warped horizon can be designed, by using a Taylor expansion along the extra dimension. Such procedure provides information about all the bulk metric components
[15]. Indeed, the bulk spacetime may be either given, by solving the full 5D equations or alternatively obtained by evolving the brane-world black hole metric off the brane, what encompasses the imprint from the bulk via the Weyl tensor. Numerical methods have been employed to find black hole solutions in the context of black strings and fluid/gravity correspondence [41]. Similar methods involving expansions of the metric have been used in the context of black strings [44], disposing the black string metric as the leading order solution in a Taylor expansion.

The bulk shape of the black string horizon has been merely investigated in very particular cases [4, 35], and latterly the standard (Schwarzschild) black string was studied in the context of a brane-world with variable tension [15]. Moreover, realistic models that take into account a post-Newtonian parameter on the Casadio-Fabbri-Mazzacurati black string [45] and the black string in a Friedmann-Robertson-Walker Eötvös brane-world [34] also represent interesting applications.

Recently regular black strings solutions associated with a dynamical brane-world have been obtained in the context of a variable brane tension [32]. The analysis of the $5 \mathrm{D}$ Kretschmann invariants makes us capable of attenuating the bulk physical singularities along some eras of the evolution of the Universe for the McVittie metric on an Eötvös fluid braneworld. This paper is devoted to encompass a framework with dark radiation and inhomogeneous dust. The 5D physical singularities in the bulk are shown to be inherited from the $4 \mathrm{D}$ brane-world and no additional singularity appears in the bulk, for some range of parameters in our model. Nevertheless, the bulk soft physical singularities can be unexpectedly controlled in the bulk upon time evolution, what makes a regular bulk 5D solution in most ranges of the dark radiation parameter.

This paper is organized as follows: in Section 2 the dark radiation dynamics on the brane is analyzed and reviewed. Starting with the Lemaittre-Tolman-Bondi (LTB) metric on the brane, the effective field equations for dark radiation on the brane are solved. The dynamical radiation model is shown to mimic a $4 \mathrm{D}$ cosmological constant on the brane. Both the black string solution and the bulk metric are obtained thereon. After obtaining the standard dark radiation model, a generalized framework is proposed. Both associated metrics are derived. In Section 3 the 5D bulk metric near the brane and the generalized black string are derived and studied, and in Section 4 the black string warped horizon in the context of inhomogeneous dust and generalized dark radiation is studied. Moreover, the black string physical singularities are analyzed from the Kretschmann invariants. The 5D physical singularities in the bulk reflect the $4 \mathrm{D}$ brane-world physical singularities. We analyze further the Kretschmann scalars generated by higher order derivatives of the Riemann tensor, and the respective soft physical singularities show that the bulk $5 \mathrm{D}$ solution is regular, in some ranges of the dark radiation parameter.

In order to fix the notation hereupon $\mu, \nu=0,1,2,3$ and $M, N=0,1,2,3,5$, and let $n$ be a time-like covector field normal to the brane and let $y$ be the associated Gaussian coordinate. The brane metric components $g_{\mu \nu}$ and 
the corresponding components of the bulk metric $\check{g}_{\mu \nu}$ are related by $\check{g}_{\mu \nu}=g_{\mu \nu}+n_{\mu} n_{\nu}$ [4]. With these choices we can write $g_{55}=1$ and $g_{\mu 5}=0$, and thus the $5 \mathrm{D}$ bulk metric reads

$$
\check{g}_{M N} d x^{M} d x^{N}=g_{\mu \nu}\left(x^{\alpha}, y\right) d x^{\mu} d x^{\nu}+d y^{2},
$$

where $M, N=0,1,2,3$ effectively.

The initial paradigm concerning a perturbative method for obtaining the black string solution consists in assuming the Schwarzschild form for the induced brane metric, on a RS brane-world. Subsequently a sheaf of such solutions is disposed into the extra dimension [31]

$$
\begin{aligned}
{ }^{(5)} d s^{2}= & e^{-2|y| / \ell}\left(-\left(1-\frac{2 G M}{r}\right) d t^{2}+\frac{d r^{2}}{1-2 G M / r}+r^{2} d \Omega_{2}\right) \\
& +d y^{2}
\end{aligned}
$$

where $\ell=\sqrt{-6 / \Lambda_{5}}$ is the curvature radius of the bulk $\mathrm{AdS}_{5}$, wherein the RS brane-world is embedded. Each space of constant $y$ is a $4 \mathrm{D}$ Schwarzschild spacetime with a line singularity along $r=0$ for all $y$, the well-known (Schwarzschild) black string.

As it is going to be clearer in Section 3, the areal radius of the sheaf of such solutions along the extra dimension is called the black string warp horizon that will be precisely defined in Section 3.

\section{Dark Radiation Dynamics on the Brane}

Henceforward some results concerning the dark radiation dynamics on the brane will be shortly revisited [46-48], in order to briefly introduce the framework to get both the bulk metric near the brane and the black string encompassing the dark radiation parameter and the effective cosmological constant. New black strings solutions are here derived in the scenario provided in [46-48] on a background described by a Lemaître-Tolman-Bondi (LTB) metric [49-51]. A solution for the black string that has as limit a tidal Reissner-Nordström black hole solution on the brane was obtained in [46], in the Randall-Sundrum scenario. In order to work with the effective Einstein equations on the brane, some conditions on the projected Weyl tensor are usually assumed, in order to provide a closed system. Besides, by taking into account a system of equations where a specific state equation leads to a inhomogeneous density that has precisely the dark radiation form [52-54] (and its generalizations for thick branes [13, 55]) and by solving the effective Einstein equations, the LTB metric can be derived. Subsequently both the bulk near the brane and the black string solution are obtained in this context.

From the dynamics of a brane-world with matter fields present in the bulk [47], the associated black string solution will be shown to present a generalized dark radiation form. The way to obtain the LTB metric is essentially different from that acquired in [46]. The inhomogeneous density is associated with conformal bulk fields, instead of being related to the electric part of the Weyl tensor. The black string solution is similarly obtained by a change in the coordinate system and its final form generalizes the first case. Thereafter two new black string solutions will be presented and subsequently used in the construction of the horizon profile in the bulk.

2.1. The Lemaître-Tolman-Bondi Metric. The Lemaître-Tolman-Bondi (LTB) metrics are exact solutions of the Einstein equations that describe inhomogeneous spacetimes, having dust as the source. This type of models considers inhomogeneous generalizations of the Friedmann-Robertson-Walker (FRW) metrics as the LTB metrics. An alternative approach for the LTB space-time is based on evolution equations of covariant objects, as the density, expansion scalar, electric Weyl tensor, shear tensor, and spatial curvature. The dynamics is reduced to scalar equations, and the FRW spacetime is achieved when two of these scalars associated with the shear tensor and electric Weyl tensor are zero. This formulation is based on a $1+3$ covariant description [56], which can be further applied to the LTB model $[57,58]$. In general, the applications of these models involve black holes, galaxy clusters, superclusters, cosmic voids, supernovas, and redshift drift, for instance, [59]. Initially found by Lemaitre [49], the LTB metric describes a spherically symmetric inhomogeneous fluid with anisotropic pressure cosmological constants that are present, for instance, in the Tolman model [50]. To derive the LTB solution, in comoving coordinates the general form for the line element is given by

$$
\begin{aligned}
d s_{(4)}^{2} & =g_{\mu \nu} d x^{\mu} d x^{\nu} \\
& =-d t^{2}+A^{2}(r, t) d r^{2}+R^{2}(r, t) d \Omega_{2},
\end{aligned}
$$

where the 2-sphere area element is denoted by $d \Omega_{2}$ and the energy-momentum tensor is written as $T_{v}^{\mu}=\operatorname{diag}(-\rho$, $\left.-\rho_{\Lambda},-\rho_{\Lambda},-\rho_{\Lambda}\right)$, where $\rho$ denotes the energy density, $\Lambda_{4}$ denotes the brane cosmological constant with associated energy density $\rho_{\Lambda}=\kappa_{4}^{-2} \Lambda_{4}$, and $\kappa_{4}$ is the $4 \mathrm{D}$ gravitational coupling constant. The Einstein equations, for each one of the space diagonal components, are given by the following expressions:

$$
\begin{gathered}
\frac{1}{R}\left\{2 \partial_{t}^{2} R+\frac{1}{R}\left[1+\left(\partial_{t} R\right)^{2}-\left(\frac{\partial_{r} R}{A}\right)^{2}\right]\right\}=\Lambda_{4}, \\
\frac{\partial_{t}^{2} R}{R}+\frac{\partial_{t} R}{R} \frac{\partial_{t} A}{A}+\frac{\partial_{r} R}{R} \frac{\partial_{r} A}{A^{3}}+\frac{\partial_{t}^{2} A}{A}-\frac{\partial_{r}^{2} R}{R A^{2}} \partial_{t} \partial_{r} R=\Lambda_{4}, \\
\frac{\partial_{t} A}{A} \partial_{r} R=\Lambda_{4} .
\end{gathered}
$$

The function $A(r, t)=g(r) \partial_{r} R$ satisfies (5). By setting $g(r)=$ $(1+f(r))^{-1 / 2}$, the usual form of the LTB metric is hence obtained:

$$
d s_{(4)}^{2}=-d t^{2}+\frac{\left(\partial_{r} R\right)^{2}}{1+f} d r^{2}+R^{2} d \Omega_{2},
$$

where $f(r)>-1$. The function $f$ can be interpreted as the energy density shell $f(r)=2 E(r)$. The function $g(r)$ is a 
geometric factor such that when $g(r)=1$ the spatial sections are flat. Equations (4) and (5) are not independent, leading to the expression

$$
\left(\partial_{t} R\right)^{2}=f+\frac{2 M}{R}+\frac{\Lambda_{4}}{3} R^{2},
$$

where $M=M(r)$ is an arbitrary function of integration that gives the gravitational mass within each comoving shell of coordinate radius $r$. By definition of mass in [51], one can write $2 d m / d r=k_{4}^{2} \rho A R^{2}$, which implies $M=$ $\int d_{r} m \sqrt{1+f} d r$. The nonrelativistic limit $f \ll 1$ yields $M \sim m$, and $(8)$ reads $(1 / 2)\left(\partial_{t} R\right)^{2}-M / R=f / 2$. The first term is interpreted as kinetic energy, the second stands for the Newtonian potential term, and $f$ is twice the energy of the system, when $\Lambda_{4}=0$. Hence $M$ is the relativistic generalization of the Newtonian mass. When $f$ is negligible, namely, in the nonrelativistic limit, the spatial sections are flat when $g=1$. The function $g$ provides the energy in each spatial section and thus carries the information of curvature for each section.

Finally, (8) implies that

$$
t-t_{N}(r)=\int_{0}^{R}\left(f+\frac{2 M}{R}+\frac{\Lambda_{4} R^{2}}{3}\right)^{-1 / 2} d R,
$$

where $t_{N}$ is known as the "bang time." Equation (8) can be used to classify the LTB models into three classes. When $\Lambda_{4}=$ 0 it reads

$$
\begin{gathered}
-1<f<0 \quad \text { elliptic, } \\
f=0 \text { parabolic, } \\
f>0 \quad \text { hyperbolic. }
\end{gathered}
$$

When $\Lambda_{4} \neq 0$ the potential $V(R)=2 M / R+\left(\Lambda_{4} / 3\right) R^{2}$ leads to a different classification, depending on the sign of $\Lambda_{4}$.

2.2. LTB Solution on the Brane. In this subsection the LTB solution associated with dark radiation on the brane is reviewed, starting with the projected Einstein equations on the brane in vacuum. Unlike the Reissner-Nordström black hole, this new solution has a specific dark radiation tidal charge $Q$. The $4 \mathrm{D}$ and 5D coupling constants are related by $\kappa_{4}^{2}=(1 / 6) \lambda \kappa_{5}^{4}$. The field equations in 5D Einstein theory lead to the projected equations $[42,60]$

$$
G_{\mu \nu}=-\Lambda_{4} g_{\mu \nu}+\kappa_{4}^{2} T_{\mu \nu}+\kappa_{5}^{4} \Pi_{\mu \nu}-\mathscr{E}_{\mu \nu}
$$

where $\Pi_{\mu \nu}$ is a term quadratic in the energy-momentum $T_{\mu \nu}$ and provides high-energy corrections arising from the extrinsic curvature of the brane, what increases the pressure and effective density of collapsing matter. The term $\mathscr{E}_{\mu \nu}$ is the projection of the bulk Weyl tensor and provides Kaluza-Klein corrections originated from 5D graviton stresses [28], as the massive modes for the graviton in the linearized regime. For observers on the brane such stresses are nonlocal, in the sense that they are local density inhomogeneities on the brane generate Weyl curvature in the bulk backreaction nonlocally on the brane $[4,7,16,17,53,54]$. Therefore the vacuum equations are $G_{\mu \nu}=-\Lambda_{4} g_{\mu \nu}-\mathscr{E}_{\mu \nu}$, where the Weyl projected tensor can be identified with a trace-free energy-momentum as $\mathscr{E}_{\mu \nu} \sim \kappa_{4}^{2} T_{\mu \nu}$, provided by [61]

$$
\mathscr{E}_{\mu \nu}=\left(\frac{\kappa_{5}}{\kappa_{4}}\right)^{4}\left[U\left(v_{\mu} v_{\nu}+\frac{1}{3} h_{\mu \nu}\right)+P_{\mu \nu}+2 Q_{(\mu} v_{\nu)}\right]
$$

where $U$ is the effective energy, $P_{\mu \nu}$ is an anisotropic stress tensor, $Q_{\mu}$ is the effective energy flux, $v_{\mu}$ is a $4 \mathrm{D}$ velocity vector satisfying $v_{\mu} v^{\mu}=-1$, and $h_{\mu \nu}$ is such that $v^{\mu} h_{\mu \nu}=0$, being thus possible to write $h_{\mu \nu}=g_{\mu \nu}+v_{\mu} v_{\nu}$. A nonstatic spherically symmetric brane-world with $P_{\mu \nu} \neq 0$ can be described by the line element

$$
d s_{(4)}^{2}=-C^{2}(r, t) d t^{2}+B^{2}(r, t) d r^{2}+R^{2}(r, t) d \Omega_{2}
$$

with $Q_{\mu}=0$. The anisotropic stress tensor $P_{\mu \nu}$ can be represented by $P_{\mu \nu}=P\left(r_{\mu} r_{\nu}+(1 / 3) h_{\mu \nu}\right)$, where $P=P(r, t)$ is a scalar field and $r_{\mu}$ is the unit radial vector. With these assumptions the electric part of the Weyl tensor yields

$$
\mathscr{E}_{\mu}^{\nu}=\left(\frac{\kappa_{5}}{\kappa_{4}}\right)^{4} \operatorname{diag}\left(\rho,-p_{r},-p_{T},-p_{T}\right)
$$

with $\rho=U, 3 p_{r}=U+2 P$, and $3 p_{T}=U-P$.

By assuming the brane field equation $\nabla^{\mu} \mathscr{E}_{\mu \nu}=0[4]$ and by considering the state equation $\rho=-p_{r}$, it yields $\partial_{t} U+$ $4\left(\partial_{t} R / R\right) U=0=\partial_{r} U+4\left(\partial_{r} R / R\right) U[46]$, implying that

$$
U=\left(\frac{\kappa_{5}}{\kappa_{4}}\right)^{4} \frac{Q}{R^{4}}
$$

where the constant $Q$ is the dark radiation tidal charge [4648]. As $\rho=U$, the energy density in this case is related to the inhomogeneous density. Thus the 4D Einstein equations (11) read

$$
G_{\mu \nu}=-\Lambda_{4} g_{\mu \nu}-\frac{Q}{R^{4}}\left(v_{\mu} v_{\nu}-2 r_{\mu} r_{\nu}+h_{\mu \nu}\right)
$$

It follows, by solving the above equations, that the component $G_{t r}$ is obtained by the expression $\partial_{t} B \partial_{r} R-B \partial_{t} \partial_{r} R=0$, and therefore the function

$$
B=H^{-1} \partial_{r} R
$$

satisfies this relation with $H=H(r)$. Considering thus such expression for $B$ in the trace equation $-G_{t}^{t}+G_{r}^{r}+2 G_{\theta}^{\theta}[46]$ it is possible to write the expression as follows:

$$
\left(\partial_{t} R\right)^{2}=f-\frac{Q}{R^{2}}+\frac{\Lambda_{4}}{3} R^{2}
$$

which is similar to (8). Integrating (18), it reads

$$
\pm t+\tau(r)=\int\left(f-\frac{Q}{R^{2}}+\frac{\Lambda_{4} R^{2}}{3}\right)^{-1 / 2} d R,
$$


which is analogous to (9). It is thus possible to write (13) in the LTB form given by (7). Making the transformation of the LTB coordinates $(t, r)$ to curvature coordinates $(T, R)$ as

$$
T=t+\int \frac{R \sqrt{\Lambda_{4} / 3-Q}}{\left(\Lambda_{4} / 3\right) R^{4}-R^{2}-Q} d R
$$

the following $4 \mathrm{D}$ metric is finally obtained:

$$
\begin{aligned}
d s_{(4)}^{2}= & -\left(1+\frac{Q}{R^{2}}-\frac{\Lambda_{4}}{3} R^{2}\right) d T^{2}+\left(1+\frac{Q}{R^{2}}-\frac{\Lambda_{4}}{3} R^{2}\right)^{-1} d R \\
& +R^{2} d \Omega_{2} .
\end{aligned}
$$

This metric is known as the inhomogeneous static exterior of a collapsing sphere of homogeneous dark radiation [28, 62]. Note that when $\Lambda_{4}=0$, the solution (21) is formally analogous to the Reissner-Nordström solution, when one identifies the electric charge to the dark radiation tidal charge.

In what follows this solution will be generalized, by considering a generalized dark radiation term with dark radiation charge $Q_{\eta}$ where $\eta$ is a parameter characterizing the model of the dark radiation [47]. The dynamics of a spherically symmetric brane-world is also analyzed, when the bulk (a) carries matter fields and (b) when its warp factor characterizes a global conformal transformation consistent with $\mathbb{Z}_{2}$ symmetry. Finally, it is possible to study the bulk metric and the black string solutions with a term analogous to the black hole solution with cosmological constant on the brane. In this framework, the energy-momentum tensor encompasses conformal bulk matter fields, whose dynamics provide a specific state equation [46-48].

Consider now a general conformal spherically symmetric metric $d s_{5}^{2}$ consistent with $\mathbb{Z}_{2}$ symmetry along the extra dimension on the brane

$$
d \dot{s}_{5}^{2}=\Omega^{2}\left(-e^{2 A} d t^{2}+e^{2 B} d r^{2}+R^{2} d \Omega_{2}+d z^{2}\right),
$$

where $z$ stands for the conformal extra dimensional coordinate and $A, B, R$, and $\Omega$ are general functions of the coordinates $(t, r, z)$. $\Omega$ denotes the conformal factor. The Einstein field equations are given by

$$
\stackrel{\circ}{G}_{M N}=-\kappa_{5}^{2}\left[-\stackrel{\circ}{T}_{M N}+\lambda \delta\left(z-z_{0}\right) \stackrel{\circ}{g}_{M N}+\Lambda_{5} \delta_{M N}\right],
$$

where $\stackrel{\circ}{g}_{M N}$ denotes the components of the the induced metric and $\stackrel{\circ}{T}_{\mu \nu}$ stands for the components of the energy-momentum tensor representing the bulk fields. The brane is localized at $z=z_{0}$.

Under the conformal transformation $\stackrel{\circ}{T}^{M N}=\Omega^{s} T^{M N}$, the energy-momentum tensor is assumed, as usual, to have weight $s=-4$ [46]. The conformal Einstein tensor is given by

$$
\stackrel{\circ}{G}_{M N}=G_{M N}+\Upsilon_{M N}(\check{g}) \text {. }
$$

By using the expression $\stackrel{\circ}{g}_{N}^{M}=\Omega^{-1} g_{N}^{M}$, (23) hence leads to the following equations:

$$
\begin{aligned}
& G_{N}^{M}=\kappa_{5}^{2} T_{N}^{M}, \\
& \Upsilon_{N}^{M}=-\kappa_{5}^{2} \Omega^{2}\left[\Omega^{-1} \lambda \delta\left(z-z_{0}\right) g_{N}^{M}+\Lambda_{5} \delta_{N}^{M}\right] .
\end{aligned}
$$

Equation (25) evinces that the 5D Einstein tensor is related solely to the presence of fields in the bulk and is independent of both the brane tension $\lambda$ and the bulk cosmological constant $\Lambda_{5}$. Moreover, (26) provides the dynamics of the conformal factor. The divergence condition leads to $\nabla_{M} T_{N}^{M}+$ $\Omega^{-1}\left[3 T_{N}^{M} \partial_{M} \Omega-T \partial_{N} \Omega\right]=0$, and from the Bianchi identity equations (25) and (26) it implies that

$$
\begin{aligned}
& \text { either } \quad \nabla_{M} T_{N}^{M}=0, \\
& \text { or } 3 T_{N}^{M} \partial_{M} \Omega=T \partial_{N} \Omega .
\end{aligned}
$$

Hence (27) require that $2 T_{z}^{z}=T_{\mu}^{\mu}$. Now, by considering the energy-momentum tensor

$$
T_{N}^{M}=\operatorname{diag}\left(-\rho, p_{r}, p_{T}, p_{T}, p_{z}\right)
$$

the state equation $\rho-p_{r}-2 p_{T}+2 p_{z}=0$ holds. It implies that $\nabla_{z} T_{z}^{z}=0$ and subsequently that $\partial_{z} p_{z}=0$. Thus $\rho, p_{r}$, and $p_{T}$ must be independent of $z$.

The system of inhomogeneous dark radiation and an effective cosmological constant is defined by a conformal bulk matter with the following equations of state:

$$
\rho=-p_{r}=\rho_{D R}+\frac{\Lambda}{\kappa_{5}^{2}}, \quad p_{T}+\eta \rho=\frac{\Lambda}{\kappa_{5}^{2}}(\eta-1),
$$

where $\eta$ characterizes the dark radiation model and $\Lambda$ is a bulk quantity and mimics a $4 \mathrm{D}$ cosmological constant on the brane. The components of the Einstein tensor can be thus evinced:

$$
\begin{gathered}
G_{r}^{r}=G_{t}^{t}=-\kappa_{5}^{2} \rho_{D R}-\Lambda, \quad G_{\theta}^{\theta}=G_{\phi}^{\phi}=-\kappa_{5}^{2} \eta \rho_{D R}-\Lambda, \\
G_{z}^{z}=-\kappa_{5}^{2} \rho_{D R}(1+\eta)-2 \Lambda .
\end{gathered}
$$

By taking the divergence of $T_{\nu}^{\mu}$, it reads

$$
\partial_{t} \rho_{D R}+2 \rho_{D R} \frac{\partial_{t} R}{R}(1-\eta)=0=\partial_{r} \rho_{D R}+2 \rho_{D R} \frac{\partial_{r} R}{R}(1-\eta),
$$

what consequently leads to

$$
\rho_{D R}=\frac{Q_{\eta}}{\kappa_{5}^{2}} R^{2 \eta-2}
$$

where here $Q_{\eta}=$ const is interpreted as a generalized dark radiation tidal charge and behaves like (15) when $\eta=-1$. Regarding the following energy conditions [63]:

$$
\begin{gathered}
\rho \geq 0, \quad \rho+p_{i} \geq 0, \\
\rho+\sum_{i=1}^{n} p_{i} \geq 0, \quad \rho+p_{i} \geq 0, \\
\rho \geq\left|p_{i}\right|,
\end{gathered}
$$

respectively, known as weak, strong, and dominant energy conditions, for $\rho=0$ (by taking the weak energy condition) 
the equality $\rho_{D R}=-\kappa_{5}^{-2} \Lambda$ holds, such that $\Lambda=-Q_{\eta} R^{2 \eta-2} \leq 0$ for $Q_{\eta} \geq 0$.

One can realize that $\rho=-p_{r}$ regards the weak condition and $\rho+p_{T}=(1-\eta) \rho_{D R}$ holds if $\eta \leq 1$, where here $\rho>0$ satisfies the first condition. By a similar analysis in $5 \mathrm{D}$ we see that $\eta \leq 0$ (weak), $\eta \leq 0$ (4D and strong), $\eta \leq-1 / 3$ (5D and strong), $|\eta| \leq 1$ ( $4 \mathrm{D}$ and dominant), and $-2 \leq \eta \leq 0$ (5D and dominant). If $Q_{\eta}<0$, all the energy conditions are violated. Taking the trace equation and then making two integrations, it yields

$$
\left(\partial_{t} R\right)^{2}=\frac{Q_{\eta}}{2 \eta+1} R^{2 \eta}+\frac{\Lambda}{3} R^{2}+f, \quad \text { for } \eta \neq-\frac{1}{2},
$$

what generalizes (18), being identical to it when $\eta=-1$. By performing one more integration it reads

$$
\pm t+\tau=\int\left[\frac{Q_{\eta}}{2 \eta+1} R^{2 \eta}+\frac{\Lambda}{3} R^{2}+f\right]^{-1 / 2} d R,
$$

where $\pm t$ refers to expansion or collapse and $\tau$ corresponds to the evaluation of the function at $t=0$. The condition $R(0, r)=$ $r$ is taken on the hypersurface $t=0$. The radial equation leads to $H=\sqrt{1+f}$ [47], and thus the metric has the 4D LTB form. Hence the 5D conformal line element reads

$$
d \dot{s}_{(5)}^{2}=\Omega_{\mathrm{RS}}^{2}\left(-d t^{2}+\left(\partial_{r} R / H\right)^{2} d r^{2}+R^{2} d \Omega_{2}+d z^{2}\right),
$$

where $\Omega_{\mathrm{RS}}$ is the Randall-Sundrum warp factor [2]. From the dynamical dark radiation models the marginal bound $f=0$ corresponds actually to static solutions. Finally, the transformation from LTB coordinates $(t, r)$ to the curvature coordinates $(T, R)$ is given thus by

$$
T=t+\int \frac{\sqrt{\left(Q_{\eta} /(2 \eta+1)\right) R^{2 \eta}+(\Lambda / 3) R^{2}}}{\left(Q_{\eta} /(2 \eta+1)\right) R^{2 \eta}+(\Lambda / 3) R^{2}-1} d R .
$$

The black string solution is therefore obtained:

$$
\begin{aligned}
d s_{(4)}^{2}= & -\left(1-\frac{Q_{\eta}}{2 \eta+1} R^{2 \eta}-\frac{\Lambda}{3} R^{2}\right) d T^{2} \\
& +\left(1-\frac{Q_{\eta}}{2 \eta+1} R^{2 \eta}-\frac{\Lambda}{3} R^{2}\right)^{-1} d R^{2}+R^{2} d \Omega_{2} .
\end{aligned}
$$

The models that describe the inhomogeneous static exterior of a collapsing sphere of homogeneous standard dark radiation require the value $\eta=-1[28,46,62]$. In general the exterior spacetime is not static in the brane-world scenario [28]; however the collapse of a homogeneous Kaluza-Klein energy density is static and can be identified to the dark radiation. Hence in the case $\eta=-1$ the model of homogeneous dark radiation is recovered. It is worth emphasizing that the exterior is static solely when the system has tidal charge and cosmological constant, where the physical mass equals zero. When $\Lambda=0$, the zero mass limit of the tidal ReissnerNordström black hole is obtained [36]. The event horizon is determined by the solutions of the following equation:

$$
1-\frac{Q_{\eta}}{2 \eta+1} R^{2 \eta}-\frac{\Lambda}{3} R^{2}=0
$$

on the brane. Thus for $\Lambda=0$ it implies that $R_{h}^{2 \eta}=(2 \eta+1) / Q_{\eta}$. When $\Lambda \neq 0$, the exact location of the horizons can not be obtained, with exceptions for the values $\eta=-1$ and $\eta=1 / 2$. Respectively, the horizons are given by

$$
\begin{aligned}
& R_{h}^{( \pm)}=\left\{\frac{3}{2 \Lambda}\left[1 \pm\left(1+\frac{4 Q_{-1} \Lambda}{3}\right)^{1 / 2}\right]\right\}^{1 / 2}, \quad \text { for } \eta=-1 \\
& R_{h}^{( \pm)}=\frac{3 Q_{1 / 2}}{4 \Lambda}\left[-1 \pm\left(1+\frac{16 \Lambda}{9 Q_{1 / 2}^{2}}\right)^{1 / 2}\right], \quad \text { for } \eta=\frac{1}{2}
\end{aligned}
$$

The conditions $\Lambda<0$ and $Q_{1 / 2}>0$ imply two horizons, an inner and an outer one. For two specific values of $Q_{1 / 2}$ the singularity is naked, thus violating the cosmic censorship hypothesis [64]. Yet, if $\Lambda>0$ and $Q_{1 / 2} \gtrless 0$, then there is a single horizon $R_{h}^{( \pm)}$.

\section{Bulk Metric and the Black String}

In this section, the bulk metric near the brane as well as the black string associated with a black hole on a brane-world is briefly introduced $[4,15,42]$. Equation (2) represents the black string metric. By denoting ${ }^{(5)} \mathscr{R}_{\mu \nu \sigma \rho}$ the components of the 5D Riemann tensor, as the 5D Kretschmann invariant ${ }^{(5)} \mathscr{R}_{\alpha \beta \rho \sigma}{ }^{(5)} \mathscr{R}^{\alpha \beta \rho \sigma}=40 / \ell^{4}+\left(48 G^{2} M^{2} / r^{6}\right) e^{4|y| / \ell}$ is unbounded as $y \rightarrow \infty[4,31]$, thus the Schwarzschild solution is not a good candidate neither for a brane-world black hole nor for trying to remove at least some of the bulk singularities. Hence a well-established perturbative method is employed to find both the bulk metric near the brane and, in particular, the black string warped horizon along the extra dimension. In what follows such framework is revisited. This will be further accomplished in Section 4 in the context of inhomogeneous dust and generalized dark radiation on the brane-world and inhomogeneous dark radiation in the bulk as well. There we will evince that in such scenario the bulk (and the black string) can be regular for certain ranges of the dark radiation parameters.

In brane-worlds with $\mathbb{Z}_{2}$ symmetry, the junction conditions imply that the extrinsic curvature of the brane is given by $[4]$

$$
K_{\mu \nu}=-\frac{\kappa_{5}^{2}}{2}\left[T_{\mu \nu}+\frac{1}{3}(\lambda-T) g_{\mu \nu}\right] .
$$

The trace-free and symmetric components of the bulk Weyl tensor $C_{\mu \nu \sigma \rho}$ are, respectively, given by $\mathscr{B}_{\mu \nu \alpha}=g_{\mu}^{\rho} g_{\nu}^{\sigma} C_{\rho \sigma \alpha \beta} n^{\beta}$ and $\mathscr{E}_{\mu \nu}=C_{\mu \nu \sigma \rho} n^{\sigma} n^{\rho}$, where $n^{\alpha}$ denote components of a vector field out of the brane-world. Hereupon we denote by $\mathscr{R}_{\mu \nu \sigma \rho}={ }^{(5)} \mathscr{R}_{\mu \nu \sigma \rho}\left(x^{\alpha}, 0\right)$ the components of the 5D Riemann tensor computed on the brane.

The effective field equations encompass the 5D Bianchi and Einstein equations [4, 23, 42], which have been generalized in a variable brane tension framework as well $[14,15$, 
$19,32,65]$. The effective field equations are provided by the following system of equations:

$$
\begin{aligned}
£ K_{\mu \nu}= & -\frac{1}{6} \Lambda_{5} g_{\mu \nu}-\mathscr{E}_{\mu \nu}+K_{\mu \rho} K_{\nu}^{\rho}, \\
\mathfrak{E} \mathscr{E}_{\mu \nu}= & \frac{1}{6} \Lambda_{5}\left(K_{\mu \nu}-K g_{\mu \nu}\right)+\nabla^{\tau} \mathscr{B}_{\tau(\mu \nu)}+\mathscr{R}_{\mu \rho \nu \sigma} K^{\rho \sigma} \\
& +K_{\rho[\mu} K_{\sigma] \nu} K^{\rho \sigma}+3 K_{(\mu}^{\rho} \mathscr{E}_{\nu) \rho}-K \mathscr{E}_{\mu \nu}, \\
\mathfrak{E} \mathscr{B}_{\mu \nu \rho}= & K_{\rho}^{\sigma} \mathscr{B}_{\mu \nu \sigma}-2 \nabla_{[\mu} \mathscr{E}_{\nu] \rho}-2 \mathscr{B}_{\rho \sigma[\mu} K_{\nu]}^{\sigma}, \\
\mathfrak{E} \mathscr{R}_{\mu \nu \rho \sigma}= & -2 \mathscr{R}_{\mu \nu \gamma[\rho} K_{\sigma]}^{\gamma}-\nabla_{\mu} \mathscr{B}_{\rho \sigma \nu}+\nabla_{\mu} \mathscr{B}_{\sigma \rho \nu} .
\end{aligned}
$$

They worked out the boundary condition $\mathscr{B}_{\mu \nu \rho}=2 \nabla_{[\mu} K_{\nu] \rho}$ on the brane [4]. These expressions are used to calculate the Taylor expansion of the brane-world metric along the extra dimension, what perturbatively defines the bulk metric near the brane and the black string horizon. The set of equations obtained from the 5D Einstein and Bianchi equations by Shiromizu et al. [4, 42] provides the bulk metric near the brane, given by the standard Taylor expansion along the extra dimension $y$ :

$$
\begin{aligned}
g_{\mu \nu}\left(x^{\alpha}, y\right)= & g_{\mu \nu}\left(x^{\alpha}, 0\right)+\left.\left(£ g_{\mu \nu}\left(x^{\alpha}, y\right)\right)\right|_{y=0}|y| \\
& +\left.\left(£\left(£ g_{\mu \nu}\left(x^{\alpha}, y\right)\right)\right)\right|_{y=0} \frac{|y|^{2}}{2 !} \\
& +\left.\left(£\left(£\left(£ g_{\mu \nu}\left(x^{\alpha}, y\right)\right)\right)\right)\right|_{y=0} \frac{|y|^{3}}{3 !}+\cdots \\
& +\left.\left(£^{k}\left(g_{\mu \nu}\left(x^{\alpha}, y\right)\right)\right)\right|_{y=0} \frac{|y|^{k}}{k !}+\cdots
\end{aligned}
$$

As comprehensively studied in [15], the term in the first-order $|y|$ above is immediately calculated from the definition of the extrinsic curvature $K_{\mu \nu}=(1 / 2) £ g_{\mu \nu}$ and from the junction condition (42) as well, by denoting $g_{\mu \nu}=g_{\mu \nu}\left(x^{\alpha}, 0\right)$ hereon. The term in $y^{2}$ is proportional to $£ K_{\mu \nu}$, which is given by (43). Now, the term $K_{\mu \alpha} K^{\alpha}{ }_{\nu}$ in (43) can be immediately computed by the junction condition (42). Moreover, the coefficient term of $|y|^{3}$ in (47) can be expressed as

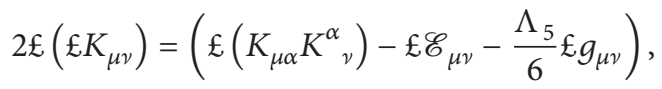

in virtue of (43). As the Lie derivatives terms in the right hand side of this last expression are, respectively, given by (43), due to (44), and by the definition of the extrinsic curvature, one hence arrives at the expression for $|y|^{3}$ in (47). Finally, the term in $y^{4}$ is acquired when the Lie derivative of the right hand side of (48) is taken into account, as well as ((43)-(46)).
Thus the above expansion up to the fourth order is given by the following formula [15]

$$
\begin{aligned}
& g_{\mu \nu}\left(x^{\alpha}, y\right) \\
& =g_{\mu \nu}-\kappa_{5}^{2}\left[T_{\mu \nu}+\frac{1}{3}(\lambda-T) g_{\mu \nu}\right]|y| \\
& +\left[\frac{1}{2} \kappa_{5}^{4}\left(T_{\mu \alpha} T_{\nu}^{\alpha}+\frac{2}{3}(\lambda-T) T_{\mu \nu}\right)\right. \\
& \left.-2 \mathscr{E}_{\mu \nu}+\frac{1}{3}\left(\frac{1}{6} \kappa_{5}^{4}(\lambda-T)^{2}-\Lambda_{5}\right) g_{\mu \nu}\right] \frac{y^{2}}{2 !} \\
& +\left[2 K_{\mu \beta} K_{\alpha}^{\beta} K_{\nu}^{\alpha}-\mathscr{E}_{(\mu \mid \alpha} K_{\mid v)}^{\alpha}-\nabla^{\rho} \mathscr{B}_{\rho(\mu \nu)}\right. \\
& +\frac{1}{6} \Lambda_{5} g_{\mu \nu} K+K^{\alpha \beta} \mathscr{R}_{\mu \alpha \nu \beta}-K \mathscr{E}_{\mu \nu} \\
& \left.+3 K_{(\mu}^{\alpha} \mathscr{E}_{\nu) \alpha}+K_{\mu \alpha} K_{\nu \beta} K^{\alpha \beta}-K^{2} K_{\mu \nu}\right] \frac{|y|^{3}}{3 !} \\
& +\left[\frac{\Lambda_{5}}{6}\left(\mathscr{R}-\frac{\Lambda_{5}}{3}+K^{2}\right) g_{\mu \nu}\right. \\
& +\left(\frac{K^{2}}{3}-\Lambda_{5}\right) K_{\mu \alpha} K^{\alpha}{ }_{\nu}+\left(\mathscr{R}-\Lambda_{5}+2 K^{2}\right) \mathscr{E}_{\mu \nu} \\
& +\left(K^{\alpha}{ }_{\tau} K^{\tau \beta}+\mathscr{E}^{\alpha \beta}+K K^{\alpha \beta}\right) \mathscr{R}_{\mu \alpha \nu \beta} \\
& +K^{2} K K_{\mu \nu}-\frac{1}{6} \Lambda_{5} R_{\mu \nu}+2 K_{\mu \beta} K_{\rho}^{\beta} K_{\alpha}^{\rho} K_{\nu}^{\alpha} \\
& +\frac{7}{2} K K_{\mu}^{\alpha} \mathscr{E}_{\nu \alpha}-\frac{7}{6} K^{\sigma \beta} K_{\mu}{ }^{\alpha} \mathscr{R}_{\nu \sigma \alpha \beta} \\
& +\mathscr{E}_{\mu \alpha}\left(\frac{1}{2} K K^{\alpha}{ }_{\nu}-3 K^{\alpha}{ }_{\sigma} K^{\alpha}{ }_{\nu}\right)-\frac{13}{2} K_{\mu \beta} \mathscr{E}_{\alpha}^{\beta}{ }_{\alpha} K^{\alpha}{ }_{\nu} \\
& \left.-4 K^{\alpha \beta} \mathscr{R}_{\mu \nu \gamma \alpha} K_{\beta}^{\gamma}-K_{\mu \alpha} K_{\nu \beta} \mathscr{E}^{\alpha \beta}\right] \frac{y^{4}}{4 !} \\
& +\cdots,
\end{aligned}
$$

where $H \equiv H_{\mu}^{\mu}$ and $H^{2} \equiv H_{\rho \sigma} H^{\rho \sigma}$, for any rank 2 tensor $H$.

The black string warped horizon [66] can be studied by the metric component $g_{\theta \theta}\left(x^{\alpha}, y\right)$ in (49). In fact, regarding a spherically symmetric $4 \mathrm{D}$ metric

$$
d s_{(4)}^{2}=-h_{1}(r) d t^{2}+h_{2}(r) d r^{2}+r^{2} d \Omega_{2},
$$

for a black hole on the brane, the usual $4 \mathrm{D}$ areal radial coordinate $r$ is related to the 5D metric by $\sqrt{g_{\theta \theta}\left(x^{\alpha}, 0\right)}=r$ $[15,35]$. The black string solution manifests a horizon on the brane with areal radius $r=\sqrt{g_{\theta \theta}\left(x^{\alpha}, 0\right)}$, where $r$ denotes the coordinate singularity usually obtained from the condition $g_{r r}^{-1}(\mathrm{r})=0$ (see equation (II.6) of reference [67]). The areal radius of the black string warped horizon is hence $\mathrm{r}(y)=$ $\left.\sqrt{g_{\theta \theta}\left(x^{\alpha}, y\right)}\right|_{r=r}$. The term $g_{\theta \theta}\left(x^{\alpha}, y\right)$ realized in (49) for $\mu=$ $\nu=\theta$ corresponds to the bulk metric near the brane and includes both the black string horizon for $r=r$, and in particular the the black hole horizon for $y=0$. 


\section{Black Strings and Dark Dust}

In this section we investigate the black string related to the induced black hole on the brane given by (38). The black string warped horizon is well known to be the component $g_{\theta \theta}\left(x^{\alpha}, y\right)$ in (49), evaluated at event horizons in (41). At first let us calculate such component for an arbitrary $x^{\alpha}$. In order to accomplish it, the $4 \mathrm{D}$ energy-momentum tensor on the brane is given by the brane-world components in (28), where

$$
\begin{gathered}
\rho=-p_{r}=\frac{Q_{\eta}}{\kappa_{5}^{2}} R^{2 \eta-2}+\frac{\Lambda}{\kappa_{5}^{2}}, \\
p_{T}=-\eta \frac{Q_{\eta}}{\kappa_{5}^{2}} R^{2 \eta-2}-\frac{\Lambda}{\kappa_{5}^{2}},
\end{gathered}
$$

and the expressions for the energy-momentum, the projected Weyl tensor components, the extrinsic curvature, and the Riemann tensor, respectively, given, respectively, by ((A.1a)(A.1c)) in Appendix are thus obtained. Hence the component $\theta \theta$ of the metric, corresponding to the black string horizon along the bulk (49), can be written as

$$
\begin{aligned}
& g_{\theta \theta}\left(x^{\alpha}, y\right) \\
& =R^{2}-\frac{R^{2}}{3}\left[\lambda \kappa_{5}^{2}+Q_{\eta} R^{2 \eta-2}(4-\eta)+3 \Lambda\right]|y| \\
& +\frac{R^{2}}{3}\left\{Q_{\eta} R^{2 \eta-2}(1-\eta)\right. \\
& +\frac{1}{6}\left(\lambda \kappa_{5}^{2}+2\left[Q_{\eta} R^{2 \eta-2}(\eta+2)+3 \Lambda\right]\right)^{2} \\
& -\Lambda_{5}-\frac{1}{2}\left(\eta Q_{\eta} R^{2 \eta-2}+\Lambda\right) \\
& \left.\cdot\left[2 \lambda \kappa_{5}^{2}+Q_{\eta} R^{2 \eta-2}(\eta+8)+9 \Lambda\right]\right\} \frac{y^{2}}{2 !} \\
& +R^{2}\left\{\frac{1}{108}\left[Q_{\eta} R^{2 \eta-2}(\eta-4)-\lambda \kappa_{5}^{2}-3 \Lambda\right]^{3}\right. \\
& -\frac{Q_{\eta}}{18} R^{2 \eta-2}(\eta-1)\left[Q_{\eta} R^{2 \eta-2}(\eta-4)-\lambda \kappa_{5}^{2}-3 \Lambda\right] \\
& -\frac{1}{9} \Lambda_{5}\left[Q_{\eta} R^{2 \eta-2}(\eta-4)-\lambda \kappa_{5}^{2}-3 \Lambda\right] \\
& -\frac{Q_{\eta}}{18} R^{2 \eta-2}(1-\eta)\left[2 \lambda \kappa_{5}^{2}+Q_{\eta} R^{2 \eta-2}(\eta+2)\right] \\
& +Q_{\eta} R^{2 \eta-2}(\eta-1)\left[\frac{Q_{\eta}}{2 \eta+1} R^{2 \eta}(\eta-1)+1\right] \\
& +\frac{\kappa_{5}^{2}}{12} \alpha^{2}\left(1-\frac{Q_{\eta}}{2 \eta+1} R^{2 \eta}-\frac{\Lambda}{3} R^{2}\right)^{-1} \exp (-\alpha T) \\
& +\frac{Q_{\eta}}{6} R^{2(\eta-2)}(2 \eta-2)(\eta+2)(2 \eta-3)
\end{aligned}
$$

$$
\begin{aligned}
& \cdot\left(1-\frac{Q_{\eta}}{2 \eta+1} R^{2 \eta}-\frac{\Lambda}{3} R^{2}\right) \\
& +\frac{1}{12} \Lambda_{5}\left[Q_{\eta} R^{2 \eta-2} \eta-\lambda \kappa_{5}^{2}-\Lambda\right] \\
& -\frac{1}{2}\left(\frac{\eta Q_{\eta}}{2 \eta+1} R^{2 \eta-2}+\frac{\Lambda}{3}\right)\left[Q_{\eta} R^{2 \eta-2} \eta+\lambda \kappa_{5}^{2}+\Lambda\right] \\
& +\frac{Q_{\eta}}{12} R^{2 \eta-2}(1-\eta)\left[Q_{\eta} R^{2 \eta-2}(\eta-4)-\lambda \kappa_{5}^{2}-3 \Lambda\right] \\
& +\frac{1}{216}\left[Q_{\eta} R^{2 \eta-2}(\eta-4)-\lambda \kappa_{5}^{2}-3 \Lambda\right] \\
& \left.\cdot\left[Q_{\eta} R^{2 \eta-2}(9 \eta-5)+3 \lambda \kappa_{5}^{2}-3 \Lambda\right]\right\} \frac{|y|^{3}}{3 !}+\cdots .
\end{aligned}
$$

The last expression does not explicitly include the terms of order $y^{4} / 4$ ! as they are extensive and awkward, although we will consider such terms in our subsequent analysis.

Our first analysis consists in regarding the variable $T=$ $T(r, t)$, which appears solely as the term $\exp (-\alpha T)$ at the order $|y|^{3} / 3$ ! in (52). Figure 1 shows the dependence of the component $g_{\theta \theta}\left(x^{\alpha}, y\right)$ on $T=T(r, t)$. Hence, near the brane, our results can be taken to be independent of the coordinate $T$. From Figures 2, 3, and 4 we depict the black string warped horizon $g_{\theta \theta}(R, y)$ as a function of the parameter $\eta$, for the possible values $\Lambda=0, \pm 1$ : Figure 2 illustrates the case of a positive cosmological constant $\Lambda=1$, where the dark radiation parameter $\eta$ plays an important role on the black string warped horizon profile. The bigger the value of $\eta$ is, the faster the warped horizon goes to zero along the extra dimension. In the range $1.8 \lesssim \eta \lesssim 8.7$ the black string warped horizon increases slower along the extra dimension; however it still goes to zero for higher values of the extra dimension. Figures 3 and 4 show, respectively, for $\Lambda=0$ and $\Lambda=-1$, quite different profiles, as the black string warped horizon is shown monotonically to increase along the extra dimension, as $\eta$ increases.

Now, the black string warped horizon is analyzed for an inhomogeneous static exterior of a collapsing sphere with homogeneous standard dark radiation, as a function of $Q_{\eta}$, as we have seen that when $\Lambda \neq 0$ in general it is not possible to obtain the exact location of the horizons by (39). In fact the two single exceptions are the models corresponding to $\eta=-1$ and $\eta=1 / 2$, given by (41). For fixed value of the dark radiation parameter, Figures 5 and 6 show that the generalized dark radiation tidal charge $Q_{\eta}$ also influences the black string warped horizon along the extra dimension. When $\eta=-1$, Figure 5 illustrates that the horizon goes to zero for some point along the extra dimension for $Q_{-1} \gtrsim 5.1$, while Figure 6 evinces that the horizon goes to zero for some point along the extra dimension for $Q_{1 / 2} \gtrsim 1.2$.

Besides the black string warped horizon, (49) provides more generally the bulk metric near the brane. In order to check whether the bulk is regular, we aim to study the $4 \mathrm{D}$ and the 5D Kretschmann invariants, related to the black 


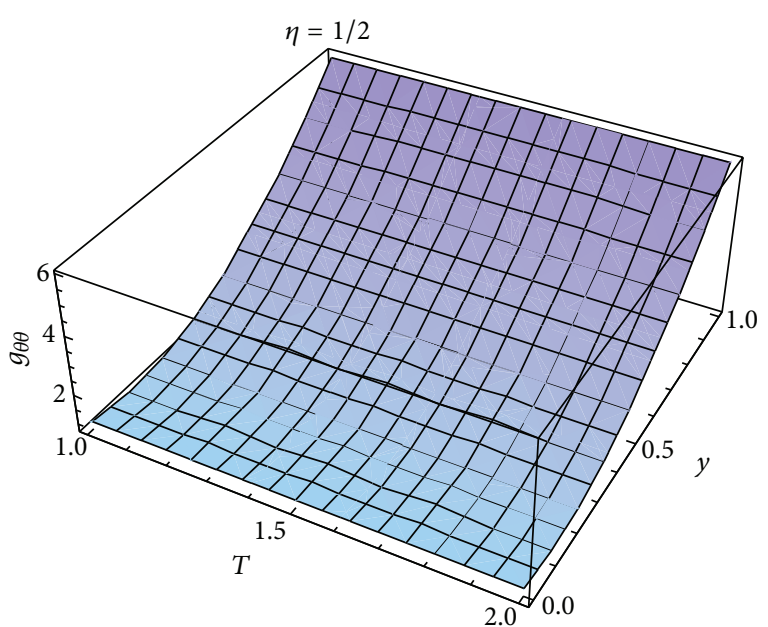

FIGURE 1: Black string warped horizon $g_{\theta \theta}(R, y)$ along the extra dimension for $\Lambda=-1$, for $\alpha=1$.

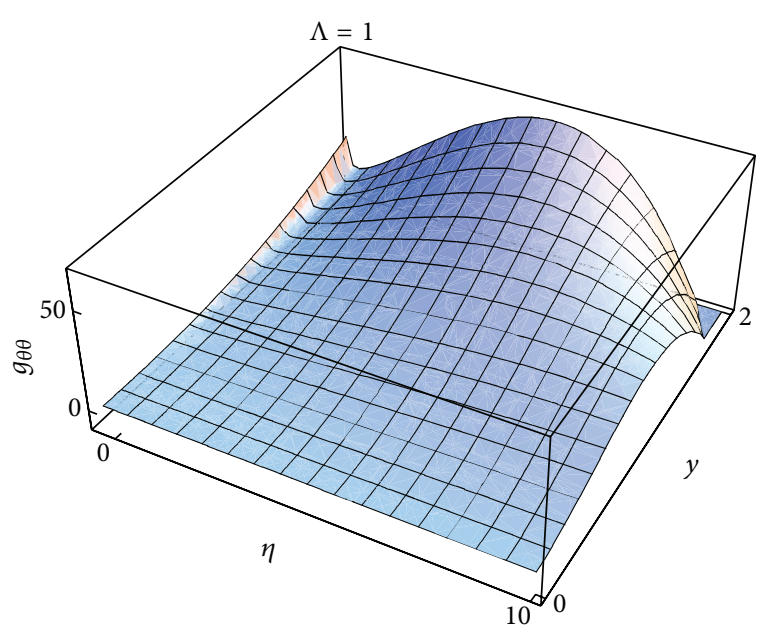

FIGURE 2: Black string warped horizon area $g_{\theta \theta}(R, y)$ along the extra dimension for $\Lambda=1$ and the parameter $\eta$ variable.

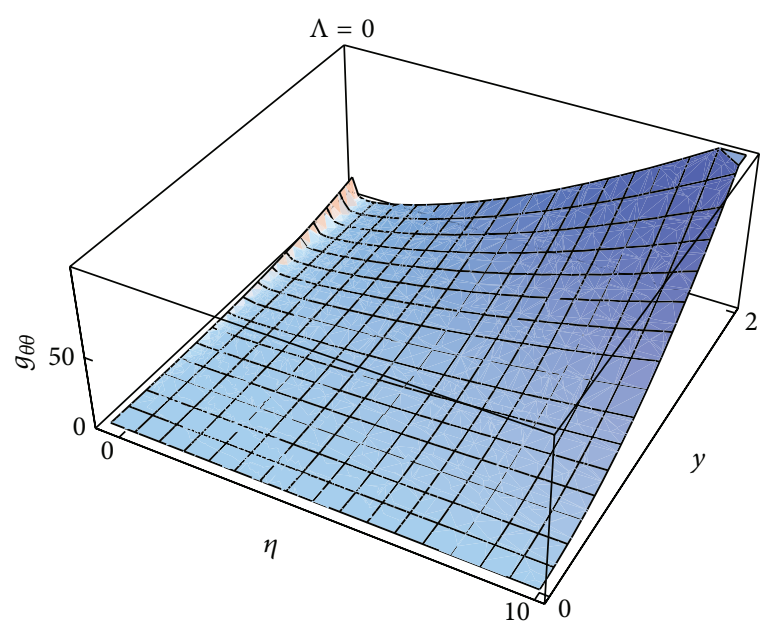

FIGURE 3: Black string warped horizon area $g_{\theta \theta}(R, y)$ along the extra dimension for $\Lambda=0$ and the parameter $\eta$ variable.

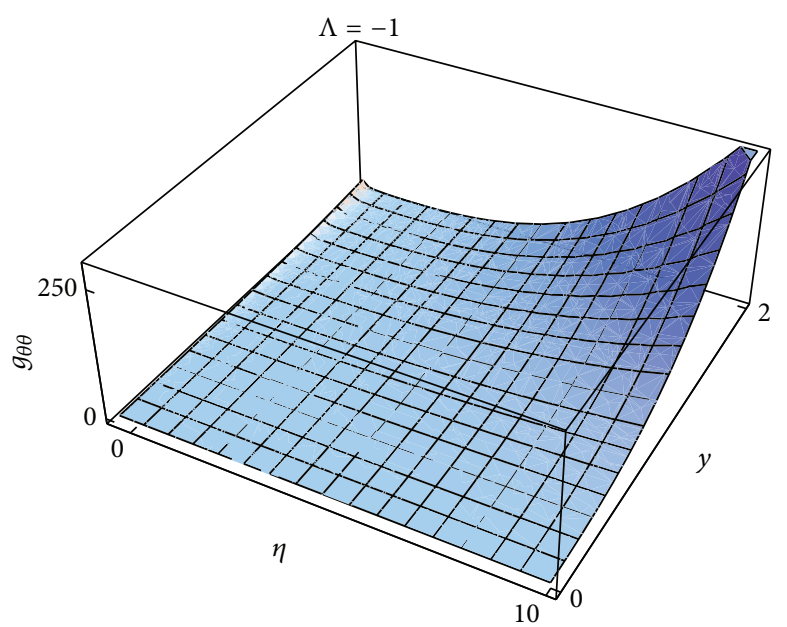

FIGURE 4: Black string warped horizon area $g_{\theta \theta}(R, y)$ along the extra dimension for $\Lambda=-1$ and the parameter $\eta$ variable.

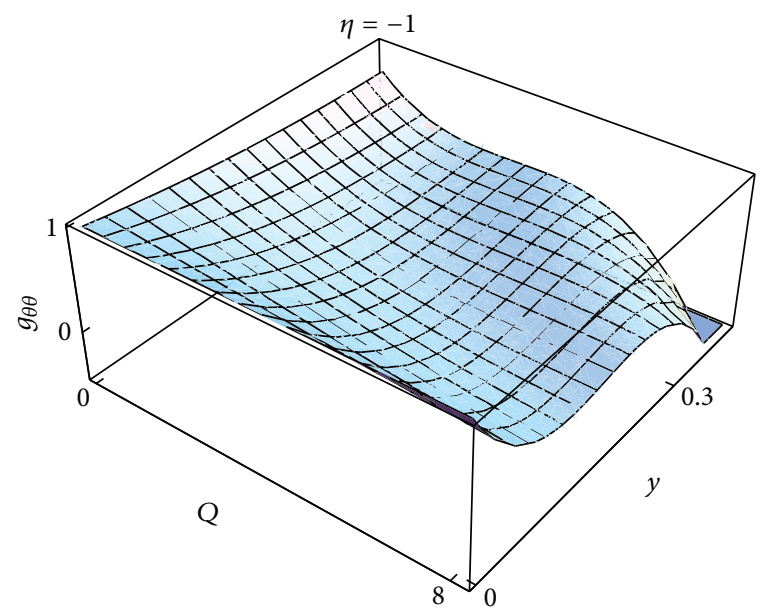

FIGURE 5: Black string warped horizon area $g_{\theta \theta}(R, y)$ along the extra dimension, as a function of the generalized dark radiation tidal charge parameter $Q_{\eta}$, for $\eta=-1$.

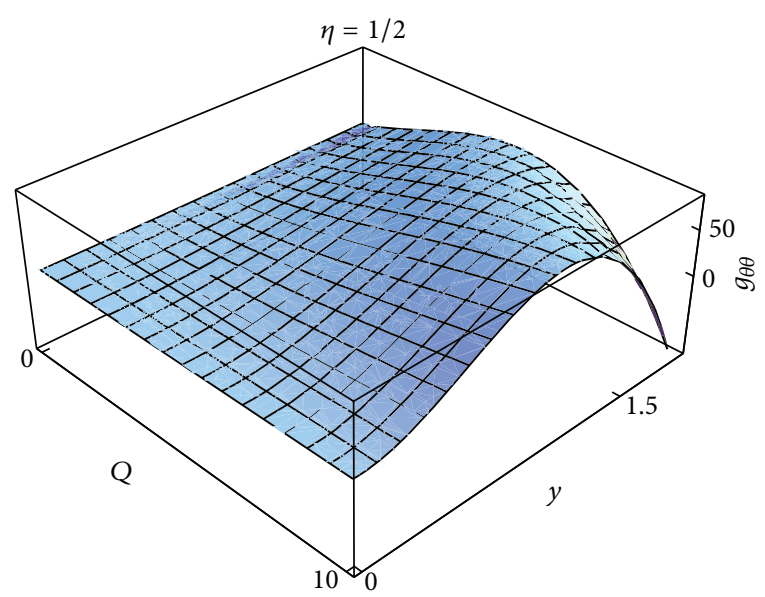

FIGURE 6: Black string warped horizon area $g_{\theta \theta}(R, y)$ along the extra dimension, as a function of $Q_{\eta}$ for $\eta=1 / 2$. 
hole on the brane and to the black string in the bulk. Our analysis of the bulk physical singularities is independent of the perturbative method (49), as the curvature invariants are independent of it.

The Gauss equation which relates the 4D and 5D Riemann curvature tensors according to

$$
{ }^{(5)} \mathscr{R}_{\nu \rho \sigma}^{\mu}={ }^{(4)} \mathscr{R}_{\nu \rho \sigma}^{\mu}+K^{\mu}{ }_{[\sigma} K_{|v| \rho]}
$$

and the $4 \mathrm{D}$ and 5D Kretschmann invariants are given by

$$
\begin{aligned}
K^{(4)} & :=\mathscr{R}^{\phi \psi \rho \sigma} \mathscr{R}_{\phi \psi \rho \sigma}, \\
K^{(5)} & :={ }^{(5)} \mathscr{R}^{\phi \psi \rho \sigma}{ }^{(5)} \mathscr{R}_{\phi \psi \rho \sigma} .
\end{aligned}
$$

For the generalized dark radiation model they are related by

$$
\begin{aligned}
K^{(5)}= & K^{(4)}-R^{2}\left[Q_{\eta} R^{2 \eta-2}(\eta-4)-\lambda \kappa_{5}^{2}-3 \Lambda\right] \\
& \cdot\left[\frac{\lambda k_{5}^{2}}{2}+Q_{\eta} R^{2 \eta-2}(\eta-1)\right] \\
\times & {\left[\frac{2}{9}\left(1-\frac{Q_{\eta} R^{2 \eta}}{2 \eta+1}-\frac{\Lambda}{3} R^{2}\right)^{-2}\left(\frac{\eta Q_{\eta} R^{2 \eta-1}}{2 \eta+1}+\frac{\Lambda}{3} R\right)\right.} \\
& \left.+\frac{1}{36 R^{4}}\left[Q_{\eta} R^{2 \eta-2}(\eta-4)-\lambda \kappa_{5}^{2}-3 \Lambda\right]\right] \\
+ & \frac{1}{18}\left(4\left[\frac{1}{2} \lambda \kappa_{5}^{2}+Q_{\eta} R^{2 \eta-2}(\eta-1)\right]^{2}\right. \\
& \left.+\left[Q_{\eta} R^{2 \eta-2}(\eta-4)-\lambda \kappa_{5}^{2}-3 \Lambda\right]^{2}\right)^{2} .
\end{aligned}
$$

The physical singularities are provided by $R=0$ and by the solutions $R_{h}$ of (39) for the values $\eta=-1$ and $\eta=1 / 2$ given by (41). Neither new singularities nor the existing ones are introduced $\left(R=0\right.$ and $\left.R=R_{h}\right)$ in (40) and (41) are removed from the bulk.

We are going to show in what follows, by the analysis of the soft physical singularities, that the bulk can be regular. Indeed, the $4 \mathrm{D}$ invariant

$$
\xi=\left(\nabla^{\mu} \nabla^{\nu} \mathscr{R}^{\phi \psi \rho \sigma}\right)\left(\nabla_{\mu} \nabla_{\nu} \mathscr{R}_{\phi \psi \rho \sigma}\right)
$$

(here $\nabla_{\mu}$ denotes the covariant derivative on the brane) is very soft, since it takes invariants involving at least two derivatives of the curvature to detect it. Consequently, the $5 \mathrm{D}$ version of the invariant $\xi$ reads

$$
{ }^{(5)} \xi=\left(D^{a} D^{b(5)} \mathscr{R}^{\phi \kappa \zeta \sigma}\right)\left(D_{a} D_{b}^{(5)} \mathscr{R}_{\phi \kappa \zeta \sigma}\right),
$$

where $D_{a}$ denotes the $5 \mathrm{D}$ covariant derivative. It is worth pointing that $a, b$ are effectively $4 \mathrm{D}$ spacetime indexes, as the $5 \mathrm{D}$ covariant derivative can be realized as $D_{a}=\nabla_{\mu}$ and $D_{a}=\nabla_{5}$, when the extra dimension $y$ is taken into account.

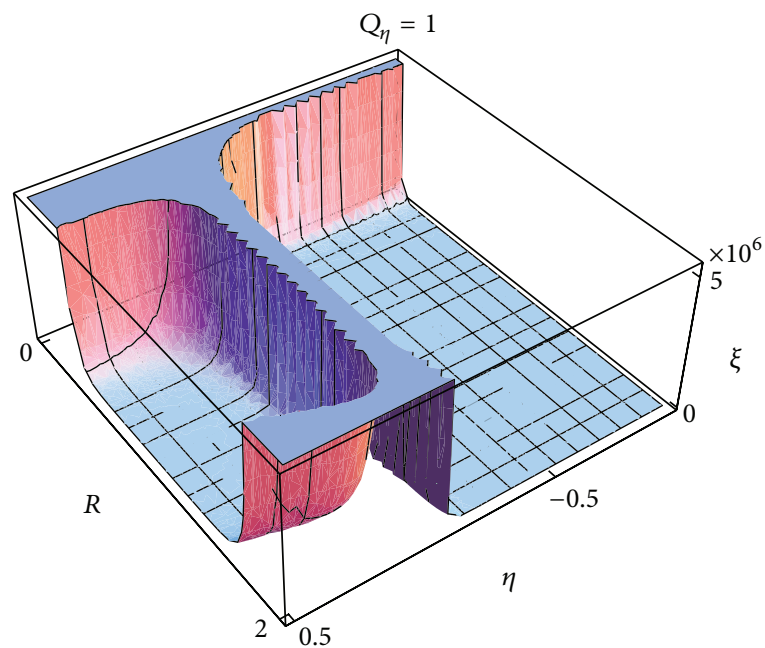

FIGURE 7: 4D Kretschmann invariant $\xi$ for $Q_{\eta}=1$, for $\Lambda=0$.

It implies that the $5 \mathrm{D}$ Kretschmann invariant ${ }^{(5)} \xi$ is given by [45]

$$
\begin{aligned}
{ }^{(5)} \xi= & \xi+2\left(\nabla_{\mu} \nabla_{\nu} K_{\tau[\rho \mid} K_{\psi \mid \sigma]}\right)\left(\nabla^{\mu} \nabla^{\nu} K^{\tau \rho} K^{\psi \sigma}\right) \\
& -2\left(\nabla_{y} \nabla_{\nu} K_{\tau \rho} K_{\psi \sigma}\right)\left(\nabla^{y} \nabla^{\nu} K^{\tau \sigma} K^{\psi \rho}\right) \\
& +\left(\nabla_{(y} \nabla_{\nu)} R_{\tau \psi \rho \sigma}\right)\left(\nabla^{y} \nabla^{\nu} R^{\tau \psi \rho \sigma}\right) \\
& -2\left(\nabla_{(\mu} \nabla_{y)} K_{\tau \rho} K_{\psi \sigma}\right)\left(\nabla^{(\mu} \nabla^{y)} R^{\tau \psi \rho \sigma}\right) \\
& -4\left(\nabla_{\mu} \nabla_{y} K_{\tau[\rho \mid} K_{\psi \mid \sigma]}\right)\left(\nabla^{\mu} \nabla^{y} K^{\tau \sigma} K^{\psi \rho}\right) \\
& +\left(\nabla_{y}^{2} R_{\tau \psi \rho \sigma}\right)\left(\left(\nabla^{y}\right)^{2} R^{\tau \psi \rho \sigma}\right) \\
& -4\left(\nabla_{y}^{2} K_{\tau \rho} K_{\psi \sigma}\right)\left(\left(\nabla^{y}\right)^{2} R^{\tau \psi \rho \sigma}\right) \\
& +2\left(\nabla_{y}^{2} K_{\tau[\sigma \mid} K_{\psi \mid \rho]}\right)\left(\left(\nabla^{y}\right)^{2} K^{\tau \sigma} K^{\psi \rho}\right) .
\end{aligned}
$$

Based on the values of the metric (38) and the extrinsic curvature components given in (42), the 5D Kretschmann invariants ${ }^{(5)} \xi$ for the bulk can calculated. Due to the awkwardness of the expression for these invariants, we opt to analyze our results by the graphics in Figures 8, 10, 12, and 14. Figure 7 has to be compared to Figure 8 that describes the $5 \mathrm{D}$ invariant ${ }^{(5)} \xi$. In Figure 7 since $\eta \sim 0$ then the $4 \mathrm{D}$ invariant $\xi$ diverges, independently of the value for $R$. On the other hand, Figure 8 depicts that, for $\eta \sim 0$, the 5D Kretschmann scale ${ }^{(5)} \xi$ goes to infinity for most values of $R$, but when $0.94 \lesssim R \lesssim 1.06$ the $5 \mathrm{D}$ Kretschmann invariant ${ }^{(5)} \xi$ does not diverge. Hence for this range of soft physical singularities present on the brane the bulk is regular. Moreover, Figure 7 shows that $\xi \rightarrow+\infty$ for $R \rightarrow 0$; however Figure 8 evinces that for $0 \leqq \eta \leqq 0.5$ the limit $\xi \rightarrow+\infty$ on the brane alters to ${ }^{(5)} \xi \rightarrow-\infty$, for values of $R \sim 0$. Still, for $-1 \lesssim \eta \lesssim-0.5$ the 5D Kretschmann invariant ${ }^{(5)} \xi$ does not diverge for $0.08 \lesssim$ $R \lesssim 0.13$, and again this range of soft physical singularities on the brane correspond to a regular bulk. Notwithstanding, 


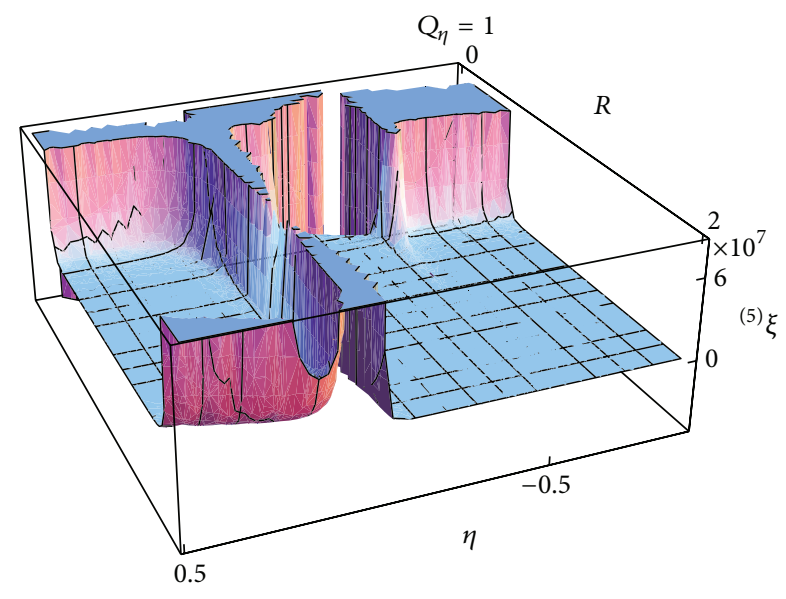

FIgURE 8: 5D Kretschmann invariant for $Q_{\eta}=1$, for $\Lambda=0$.

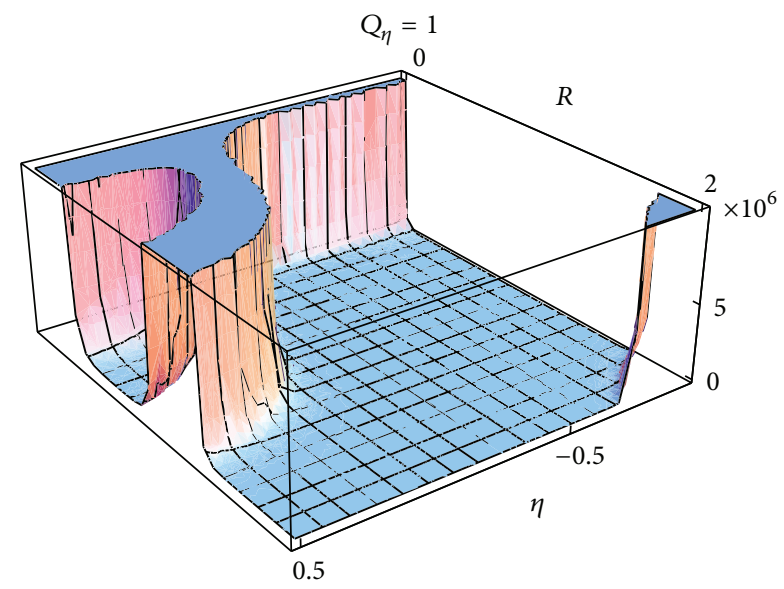

FIGURE 9: 4D Kretschmann invariant $\xi$ for $Q_{\eta}=1$, for $\Lambda=1$.

now there is a range $-1 \lesssim \eta \lesssim-0.5$ and $0.08-0.13 \lesssim R \lesssim$ $0.47-0.49$ that makes the 5D Kretschmann invariant ${ }^{(5)} \xi$ to diverge.

Now the case $\Lambda=1$ is regarded. Figure 9, regarding the $4 \mathrm{D}$ invariant $\xi$, must be compared, respectively, to Figure 10, describing the $5 \mathrm{D}$ invariant ${ }^{(5)} \xi$. A similar pattern is realized in this case, where now Figure 9 shows that $\xi \rightarrow+\infty$ for $R \rightarrow 0$. We regard the $\Lambda=-1$ case in Figures 11 and 12, and the conclusions are analogous to both previous cases. Finally, the values of the $4 \mathrm{D}$ and the $5 \mathrm{D}$ Kretschmann invariants are provided for $Q_{\eta}=10$ and $\Lambda=0$. The respective graphics for $\Lambda= \pm 1$ are quite similar.

Figure 13 illustrates that whatever the value for $\eta$ in the range $[-1,1 / 2]$ is, the $5 D$ curvature invariant ${ }^{(5)} \xi$ is finite for $R \gtrsim 0.71$, and the whole bulk is regular, independent of the value of the extra dimension $y$. Nevertheless, Figure 14 also evinces the same pattern of the previous cases. For $0 \lesssim \eta \lesssim 0.5$ the limit $\xi \rightarrow+\infty$ on the brane is modified into the bulk to (5) $\xi \rightarrow-\infty$, for values of $R$ near $R=0$. Still, again for $-1 \lesssim$ $\eta \lesssim-0.5$ the $5 \mathrm{D}$ Kretschmann invariant ${ }^{(5)} \xi$ does not diverge for $0.08 \leqslant R \leqslant 0.13$, and again this range of soft physical

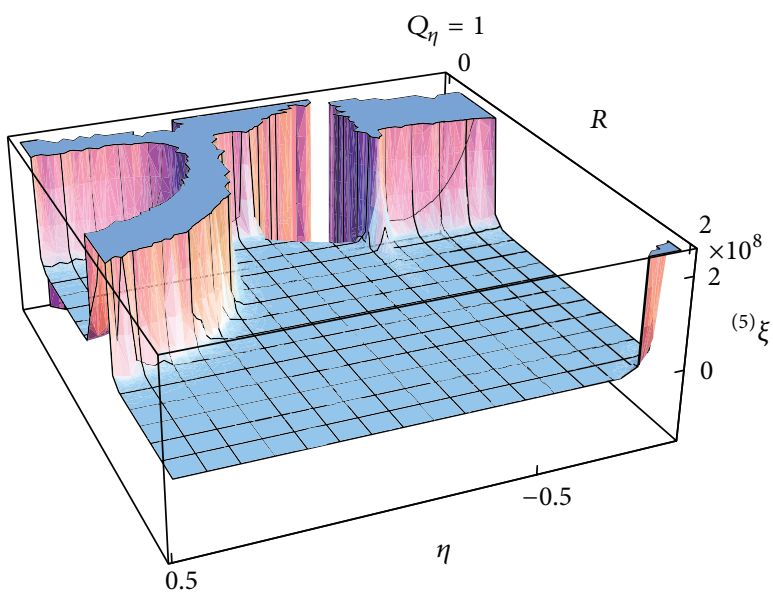

FIGURE 10: 5D Kretschmann invariant ${ }^{(5)} \xi$ for $Q_{\eta}=1$, for $\Lambda=1$.

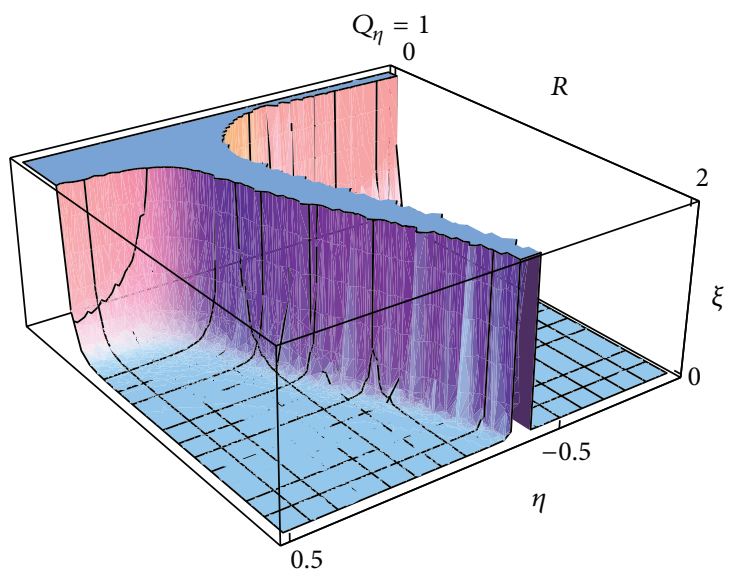

FIGURE 11: 4D Kretschmann invariant $\xi$ for $Q_{\eta}=1$, for $\Lambda=-1$.

singularities, present on the brane, is banished in the bulk, as the Kretschmann invariants remain finite from a starting finite value for $R$.

\section{Concluding Remarks}

The bulk metric near the brane was obtained and in particular the black string warped horizon for inhomogeneous dust and generalized dark radiation on the brane-world and inhomogeneous dark radiation in the bulk are regarded. The standard dark radiation $[46,52-54]$ is a particular case analyzed, where the dark radiation parameter $\eta=-1$ and the mimicked cosmological constant $\Lambda$ equal zero, provided by the $5 \mathrm{D}$ pressure also equal to zero.

By analyzing the 4D and 5D standard Kretschmann invariants, respectively, defined by (54) and (55), the Gauss equation is shown to imply that the bulk associated with brane-world models with inhomogeneous dust and generalized dark radiation inherits the brane-world physical singularities at $R=0$ and at $R=R_{h}$ is the solution of (39). Although the black string warped horizon is obtained as a particular case of the 5D bulk metric near the brane via an 


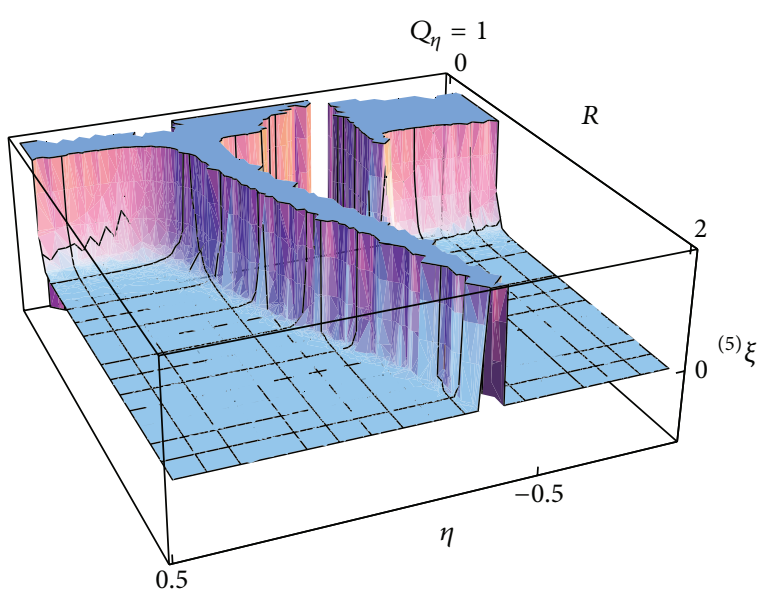

FIGURE 12: 5D Kretschmann invariant ${ }^{(5)} \xi$ for $Q_{\eta}=1$, for $\Lambda=-1$.

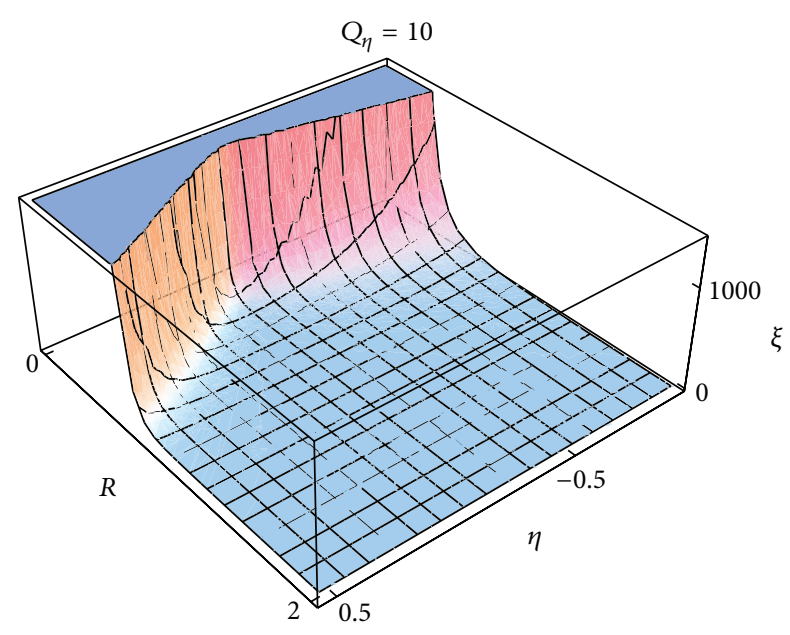

FIGURE 13: 4D Kretschmann invariant $\xi$ for $Q_{\eta}=10$, for $\Lambda=0$.

effective method, the analysis of the 5D bulk singularities relies on an exact method provided by the Gauss equation (53).

The 4D and 5D Kretschmann invariants, respectively, given by (57) and (58), show that although some soft physical singularities exist on the brane, the 5D bulk can be regular, for some parameters of the dark radiation parameter and for various values of the generalized dark radiation tidal charge parameter $Q_{\eta}$ analyzed. Figures 7-14 show these features for some values of $Q_{\eta}$ and all possible values of the mimicked cosmological constant on the brane. In fact Figures 7, 9, 11, and 13 depict the 4D Kretschmann invariants, while Figures $8,10,12$, and 14 illustrate the $5 \mathrm{D}$ ones, for all values of the mimicked $4 \mathrm{D}$ cosmological constant on the brane $\Lambda=0, \pm 1$. Such figures evince that the bulk is more regular in what concerns the respective soft singularities, for certain ranges of the generalized dark radiation tidal charge parameter analyzed.

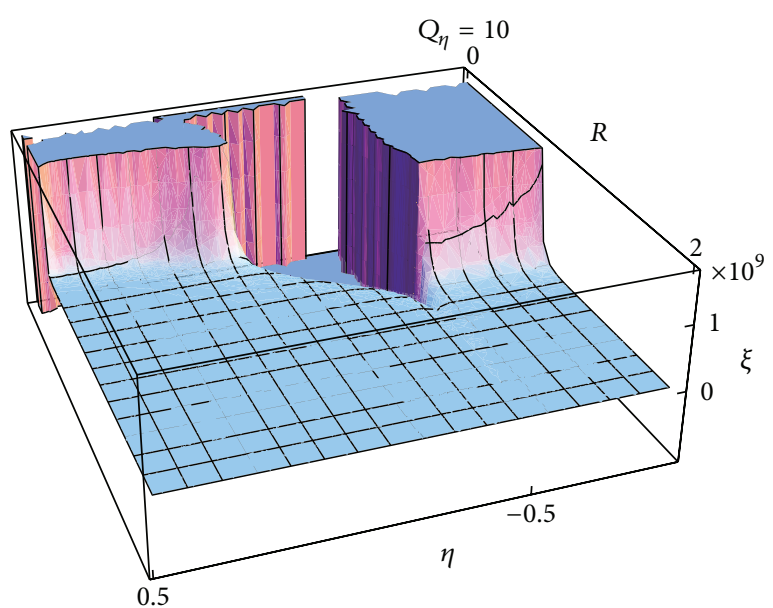

FIGURE 14: 5D Kretschmann invariant ${ }^{(5)} \xi$ for $Q_{\eta}=10$, for $\Lambda=0$.

\section{Appendix}

\section{Energy-Momentum, Weyl Tensor, and Extrinsic Curvature Components}

The components of the energy-momentum and the Weyl tensor are given, respectively, by

$$
\begin{aligned}
& T_{\theta \theta}=-\frac{R^{2}}{\kappa_{5}^{2}}\left(\eta Q_{\eta} R^{2 \eta-2}+\Lambda\right), \\
& T_{\varphi \varphi}=-\frac{R^{2}}{\kappa_{5}^{2}}\left(\eta Q_{\eta} R^{2 \eta-2}+\Lambda\right) \sin ^{2} \theta \\
& \left.T_{T T}=\frac{2}{\kappa_{5}^{2}}\left(1-\frac{Q_{\eta}}{2 \eta+1} R^{2 \eta}-\frac{\Lambda}{3} R^{2}\right)\left(Q_{\eta} R^{2 \eta-2}+\Lambda\right), \quad \text { A. } 1 \mathrm{~b}\right) \\
& T_{R R}=-\frac{2}{\kappa_{5}^{2}}\left(1-\frac{Q_{\eta}}{2 \eta+1} R^{2 \eta}-\frac{\Lambda}{3} R^{2}\right)^{-1}\left(Q_{\eta} R^{2 \eta-2}+\Lambda\right), \\
& \mathscr{E}_{\theta \theta}=-\frac{Q_{\eta}}{6}(1-\eta) R^{2 \eta}, \quad \mathscr{E}_{\varphi \varphi}=\mathscr{E}_{\theta \theta} \sin ^{2} \theta \\
& \mathscr{E}_{T T}=-\frac{Q_{\eta}}{3}(1-\eta) R^{2 \eta-2}\left(1-\frac{Q_{\eta}}{2 \eta+1} R^{2 \eta}-\frac{\Lambda}{3} R^{2}\right), \\
& \mathscr{E}_{R R}=\frac{Q_{\eta}}{3}(1-\eta) R^{2 \eta-2}\left(1-\frac{Q_{\eta}}{2 \eta+1} R^{2 \eta}-\frac{\Lambda}{3} R^{2}\right)^{-1} .
\end{aligned}
$$

For the extrinsic curvature, by computing (42) we obtain

$$
\begin{aligned}
& K_{\theta \theta}=\frac{R^{2}}{6}\left[Q_{\eta} R^{2 \eta-2}(\eta-4)-\lambda \kappa_{5}^{2}-3 \Lambda\right], \\
& K_{\varphi \varphi}=K_{\theta \theta} \sin ^{2} \theta,
\end{aligned}
$$




$$
\begin{aligned}
K_{T T}= & \frac{1}{3}\left(1-\frac{Q_{\eta}}{2 \eta+1} R^{2 \eta}-\frac{\Lambda}{3} R^{2}\right) \\
\cdot & {\left[\frac{1}{2} \lambda \kappa_{5}^{2}+Q_{\eta} R^{2 \eta-2}(\eta-1)\right], } \\
K_{R R}=- & \frac{1}{3}\left(1-\frac{Q_{\eta}}{2 \eta+1} R^{2 \eta}-\frac{\Lambda}{3} R^{2}\right)^{-1} \\
\cdot & {\left[\frac{1}{2} \lambda \kappa_{5}^{2}+Q_{\eta} R^{2 \eta-2}(\eta-1)\right] . }
\end{aligned}
$$

Finally, the necessary components of the Riemann tensor are given by

$$
\begin{aligned}
& \mathscr{R}_{\theta T \theta T}=\left(\frac{Q_{\eta}}{2 \eta+1} R^{2 \eta}+\frac{\Lambda}{3} R^{2}-1\right)\left(\frac{\eta Q_{\eta}}{2 \eta+1} R^{2 \eta}+\frac{\Lambda}{3} R^{2}\right), \\
& \text { (A.4a) } \\
& \mathscr{R}_{\theta R \theta R}=\left(1-\frac{Q_{\eta}}{2 \eta+1} R^{2 \eta}-\frac{\Lambda}{3} R^{2}\right)^{-1}\left(\frac{\eta Q_{\eta}}{2 \eta+1} R^{2 \eta}+\frac{\Lambda}{3} R^{2}\right), \\
& \text { (A.4b) } \\
& \mathscr{R}_{\theta \varphi \theta \varphi}=R^{2} \sin ^{2} \theta\left[\frac{Q_{\eta}}{2 \eta+1} R^{2 \eta}+\frac{\Lambda}{3} R^{2}\right] .
\end{aligned}
$$

\section{Conflict of Interests}

The authors declare that there is no conflict of interests regarding the publication of this paper.

\section{Acknowledgments}

A. M. Kuerten is grateful to CAPES and Programa Ciência sem Fronteiras (CsF) for financial support and to the ICF, UNAM, for hospitality. Roldão da Rocha thanks SISSA for the hospitality and is grateful to CNPq Grants no. 303027/2012-6 and no. 473326/2013-2 for partial financial support. Roldão da Rocha is also Bolsista da CAPES Proc. 10942/13-0. A. Herrera-Aguilar thanks the ICF, UNAM, and UAMIztapalapa for hospitality and is grateful as well to PAPIITUNAM, IN103413-3, Teorías de Kaluza-Klein, inflación y perturbaciones gravitacionales, and SNI for financial support.

\section{References}

[1] L. Randall and R. Sundrum, "Large mass hierarchy from a small extra dimension," Physical Review Letters, vol. 83, no. 17, pp. 3370-3373, 1999.

[2] L. Randall and R. Sundrum, "An alternative to compactification," Physical Review Letters, vol. 83, no. 23, pp. 4690-4693, 1999.

[3] P. Hořava and E. Witten, "Heterotic and type-I string dynamics from eleven dimensions," Nuclear Physics B, vol. 460, no. 3, pp. 506-524, 1996.

[4] R. Maartens and K. Koyama, "Brane-world gravity," Living Reviews in Relativity, vol. 13, no. 5, 2010.

[5] M. Gogberashvili, "Our world as an expanding shell," Europhysics Letters, vol. 49, no. 3, pp. 396-399, 2000.
[6] P. Binétruy, C. Deffayet, and D. Langlois, "Non-conventional cosmology from a brane universe," Nuclear Physics, B, vol. 565, no. 1-2, pp. 269-287, 2000.

[7] R. Maartens, "Cosmological dynamics on the brane," Physical Review D, vol. 62, no. 8, Article ID 084023, 2000.

[8] A. Campos and C. F. Sopuerta, "Evolution of cosmological models in the brane-world scenario," Physical Review D, vol. 63, no. 10, Article ID 104012, 2001.

[9] M. Gogberashvili, A. Herrera-Aguilar, D. Malagón-Morejón, R. R. Mora-Luna, and U. Nucamendi, "Thick brane isotropization in a generalized 5D anisotropic standing wave braneworld model," Physical Review D-Particles, Fields, Gravitation and Cosmology, vol. 87, no. 8, Article ID 084059, 2013.

[10] N. Barbosa-Cendejas and A. Herrera-Aguilar, "4D gravity localized in non $\mathbb{Z}_{2}$-symmetric thick branes," Journal of High Energy Physics, no. 10, article 101, 2005.

[11] J. E. Silva, V. Santos, and C. A. Almeida, "Gravity localization in a string-cigar braneworld," Classical and Quantum Gravity, vol. 30, no. 2, Article ID 025005, 2013.

[12] I. C. Jardim, R. R. Landim, G. Alencar, and R. N. Costa Filho, "Construction of multiple spherical branes cosmological scenario," Physical Review D, vol. 84, no. 6, Article ID 064019, 2011.

[13] A. E. Bernardini, R. T. Cavalcanti, and R. da Rocha, "Spherically symmetric thick branes cosmological evolution," General Relativity and Gravitation, vol. 47, no. 1, article 1840, 2015.

[14] M. C. Abdalla, J. M. Hoff da Silva, and R. da Rocha, "Notes on the two-brane model with variable tension," Physical Review D: Particles, Fields, Gravitation, and Cosmology, vol. 80, no. 4, Article ID 046003, 2009.

[15] R. da Rocha and J. M. Hoff da Silva, "Black string corrections in variable tension braneworld scenarios," Physical Review D: Particles, Fields, Gravitation and Cosmology, vol. 85, no. 4, Article ID 046009, 2012.

[16] M. Gogberashvili, A. Herrera-Aguilar, and D. MalagónMorejón, "An anisotropic standing wave braneworld and associated Sturm-Liouville problem," Classical and Quantum Gravity, vol. 29, no. 2, Article ID 025007, 2012.

[17] M. Gogberashvili, A. Herrera-Aguilar, D. Malagón-Morejón, and R. R. Mora-Luna, "Anisotropic inflation in a 5D standing wave braneworld and effective dimensional reduction," Physics Letters B, vol. 725, no. 4-5, pp. 208-211, 2013.

[18] J. M. Hoff da Silva and R. da Rocha, "Braneworld remarks in Riemann-Cartan manifolds," Classical and Quantum Gravity, vol. 26, no. 5, Article ID 055007, 9 pages, 2009.

[19] R. Casadio, J. Ovalle, and R. da Rocha, "Black strings from minimal geometric deformation in a variable tension braneworld," Classical and Quantum Gravity, vol. 31, no. 4, Article ID 045016, 2014.

[20] J. M. H. da Silva and R. da Rocha, "Schwarzschild generalized black hole horizon and the embedding space," The European Physical Journal C, vol. 72, pp. 22-58, 2012.

[21] J. M. da Silva and R. da Rocha, "Gravitational constraints of dS branes in an AdS EInstein-Brans-Dicke bulk," Classical and Quantum Gravity, vol. 27, no. 22, Article ID 225008, 2010.

[22] C. H. Coimbra-Araújo, R. D. A. Rocha, and I. T. Pedron, "Antide sitter curvature radius constrained by quasars in brane-world scenarios," International Journal of Modern Physics D, vol. 14, no. 11, pp. 1883-1898, 2005.

[23] L. A. Gergely, “Generalized Friedmann branes," Physical Review, D: Third Series, vol. 68, no. 12, Article ID 124011, 2003. 
[24] P. Kanti, N. Pappas, and K. Zuleta, "On the localization of fourdimensional brane-world black holes," Classical and Quantum Gravity, vol. 30, no. 23, Article ID 235017, 19 pages, 2013.

[25] R. Casadio and J. Ovalle, "Brane-world stars from minimal geometric deformation, and black holes," General Relativity and Gravitation, vol. 46, article 1669, 2014.

[26] N. Dadhich and S. G. Ghosh, "Gravitational collapse of null fluid on the brane," Physics Letters B, vol. 518, no. 1-2, pp. 1-7, 2001.

[27] G. Kofinas and E. Papantonopoulos, "Gravitational collapse in braneworld models with curvature corrections," Journal of Cosmology and Astroparticle Physics, vol. 12, article 011, 2004.

[28] M. Bruni, C. Germani, and R. Maartens, "Gravitational collapse on the brane: a no-go theorem," Physical Review Letters, vol. 87, no. 23, Article ID 231302, 2001.

[29] C. G. Bohmer and T. Harko, "Galactic dark matter as a bulk effect on the brane," Classical and Quantum Gravity, vol. 24, p. 3191, 2007.

[30] G. T. Horowitz and A. Strominger, "Black strings and p-branes," Nuclear Physics B, vol. 360, no. 1, pp. 197-209, 1991.

[31] A. Chamblin, S. W. Hawking, and H. S. Reall, "Brane-world black holes," Physical Review D, vol. 61, Article ID 065007, 2000.

[32] D. Bazeia, J. M. Hoff da Silva, and R. da Rocha, "Regular bulk solutions a nd black strings from dynamical brane-worlds with variable tension," Physical Review D, vol. 90, Article ID 047902, 2014.

[33] R. Casadio, A. Fabbri, and L. Mazzacurati, "New black holes in the brane world?" Physical Review D, vol. 65, no. 8, Article ID 084040, 5 pages, 2002.

[34] R. da Rocha, A. Piloyan, A. M. Kuerten, and C. H. CoimbraAraujo, "Casadio-Fabbri-Mazzacurati black strings and braneworld-induced quasars luminosity corrections," Classical and Quantum Gravity, vol. 30, no. 4, Article ID 045014, 2013.

[35] R. Casadio and C. Germani, "Gravitational collapse and black hole evolution-do holographic black holes eventually "antievaporate"?” Progress of Theoretical Physics, vol. 114, no. 1, pp. 23-56, 2005.

[36] N. Dadhich, R. Maartens, P. Papadopoulos, and V. Rezania, "Black holes on the brane," Physics Letters B, vol. 487, no. 1-2, pp. 1-6, 2000.

[37] C. Germani and R. Maartens, "Stars in the braneworld," Physical Review D, vol. 64, no. 12, Article ID 124010, 2001.

[38] M. Visser and D. L. Wiltshire, "On-brane data for braneworld stars," Physical Review D, vol. 67, Article ID 104004, 2003.

[39] P. Kanti, I. Olasagasti, and K. Tamvakis, "Quest for localized 4D black holes in brane worlds. II. Removing the bulk singularities," Physical Review D, vol. 68, no. 12, Article ID 124001, 10 pages, 2003.

[40] R. Gregory and R. Laflamme, "Black strings and p-branes are unstable," Physical Review Letters, vol. 70, no. 19, pp. 2837-2840, 1993.

[41] M. Choptuik, L. Lehner, I. Olabarrieta, R. Petryk, F. Pretorius, and H. Villegas, "Towards the final fate of an unstable black string," Physical Review D, vol. 68, no. 4, Article ID 044001, 2003.

[42] T. Shiromizu, K. Maeda, and M. Sasaki, "The Einstein equations on the 3-brane world," Physical Review, D: Third Series, vol. 62, no. 2, Article ID 024012, 2000.

[43] L. A. Gergely, "Black holes and dark energy from gravitational collapse on the brane," Journal of Cosmology and Astroparticle Physics, vol. 2, article 27, 2007.
[44] N. Haddad, "Black strings ending in horizons," Classical and Quantum Gravity, vol. 29, Article ID 245001, 2012.

[45] D. Bazeia, J. M. H. da Silva, and R. da Rocha, "Black holes in realistic branes: black string-like objects?” Physics Letters B, vol. 721, no. 4-5, pp. 306-311, 2013.

[46] R. Neves and C. Vaz, "Dark radiation dynamics on the brane," Physical Review D, vol. 66, no. 12, Article ID 124002, 2002.

[47] R. Neves and C. Vaz, "Brane world dynamics and conformal bulk fields," Physical Review D, vol. 68, Article ID 024007, 2003.

[48] R. Neves, "Braneworlds, conformal fields and the gravitons," Journal of Physics A: Mathematical and Theoretical, vol. 40, no. 25, pp. 6991-6997, 2007.

[49] A. G. Lemaître, "L’Univers en Expansion," General Relativity and Gravitation, vol. 29, no. 5, pp. 641-680, 1997.

[50] R. C. Tolman, "Effect of inhomogeneity on cosmological models," Proceedings of the National Academy of Sciences of the United States of America, vol. 20, no. 3, pp. 169-176, 1934.

[51] H. Bondi, "Spherically symmetrical models in general relativity," Royal Astronomical Society. Monthly Notices, vol. 107, pp. 410-425, 1947.

[52] P. Binétruy, C. Deffayet, U. Ellwanger, and D. Langlois, "Brane cosmological evolution in a bulk with cosmological constant," Physics Letters B: Nuclear, Elementary Particle and High-Energy Physics, vol. 477, no. 1-3, pp. 285-291, 2000.

[53] S. Mukohyama, "Brane-world solutions, standard cosmology, and dark radiation," Physics Letters B, vol. 473, no. 3-4, pp. 241245, 2000.

[54] P. Bowcock, C. Charmousis, and R. Gregory, "General brane cosmologies and their global spacetime structure," Classical and Quantum Gravity, vol. 17, no. 22, pp. 4745-4763, 2000.

[55] A. Ahmed, L. Dulny, and B. Grzadkowski, "Generalized Randall-Sundrum model with a single thick brane," The European Physical Journal C, vol. 74, no. 4, pp. 28-62, 2014.

[56] G. F. R. Ellis and H. van Elst, "Cosmological models (Cargèse lectures 1998)," http://arxiv.org/abs/gr-qc/9812046.

[57] R. A. Sussman, "A dynamical system approach to inhomogeneous dust solutions," Classical and Quantum Gravity, vol. 25, no. 1, Article ID 015012, 2008.

[58] R. A. Sussman, "Quasilocal variables in spherical symmetry: numerical applications to dark matter and dark energy sources," Physical Review D: Particles, Fields, Gravitation and Cosmology, vol. 79, Article ID 025009, 2009.

[59] K. Bolejko, A. Krasiński, C. Hellaby, and M.-N. Célérier, Structures in the Universe by Exact Methods: Formation, Evolution, Interactions, Cambridge Monographs on Mathematical Physics, Cambridge University Press, Cambridge, UK, 2010.

[60] A. N. Aliev and A. E. Gümrükçüoglu, "Gravitational field equations on and off a 3-brane world," Classical and Quantum Gravity, vol. 21, no. 22, pp. 5081-5095, 2004.

[61] R. Maartens, "Cosmological dynamics on the brane," Physical Review D, vol. 62, no. 8, Article ID 084023, 2000.

[62] M. Govender and N. Dadhich, "Collapsing sphere on the brane radiates," Physics Letters. B, vol. 538, no. 3-4, pp. 233-238, 2002.

[63] R. M. Wald, General Relativity, The University of Chicago Press, Chicago, Ill, USA, 1984.

[64] R. Penrose, "Gravitational collapse: the role of general relativity," Rivista del Nuovo Cimento, vol. 1, pp. 252-276, 1969.

[65] L. A. Gergely, "Friedmann branes with variable tension," Physical Review D, vol. 78, no. 8, Article ID 084006, 2008. 
[66] S. S. Seahra, C. Clarkson, and R. Maartens, "Detecting extra dimensions with gravity-wave spectroscopy: the black-string brane world," Physical Review Letters, vol. 94, no. 12, Article ID 121302, 2005.

[67] R. Casadio and B. Harms, "Black hole evaporation and compact extra dimensions," Physical Review D, vol. 64, no. 2, Article ID 024016, 2001. 

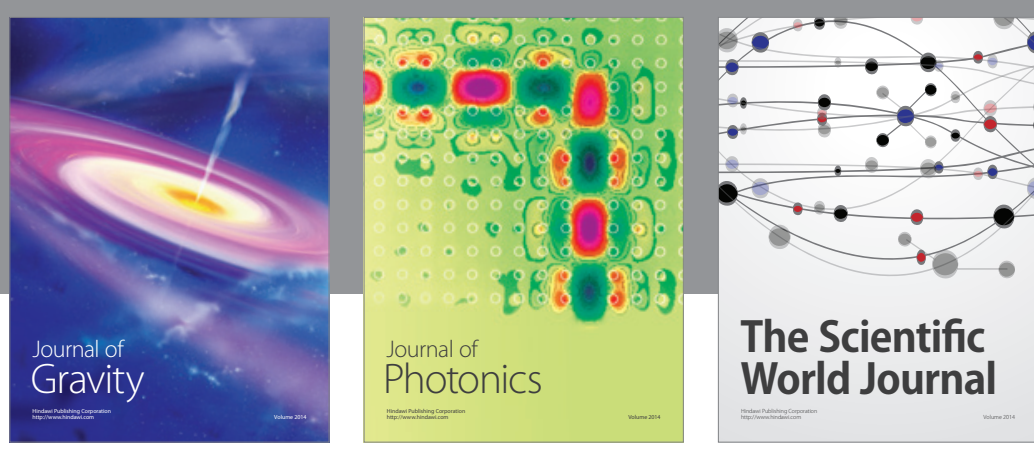

The Scientific World Journal
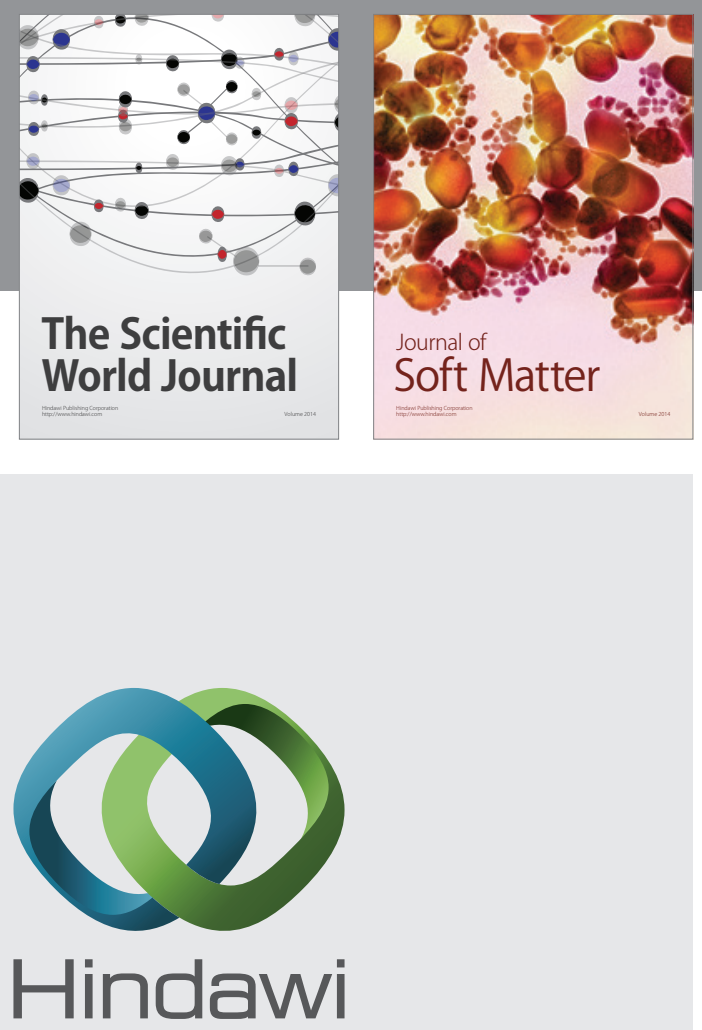

Submit your manuscripts at

http://www.hindawi.com

nternational Journal of

Statistical Mechanics
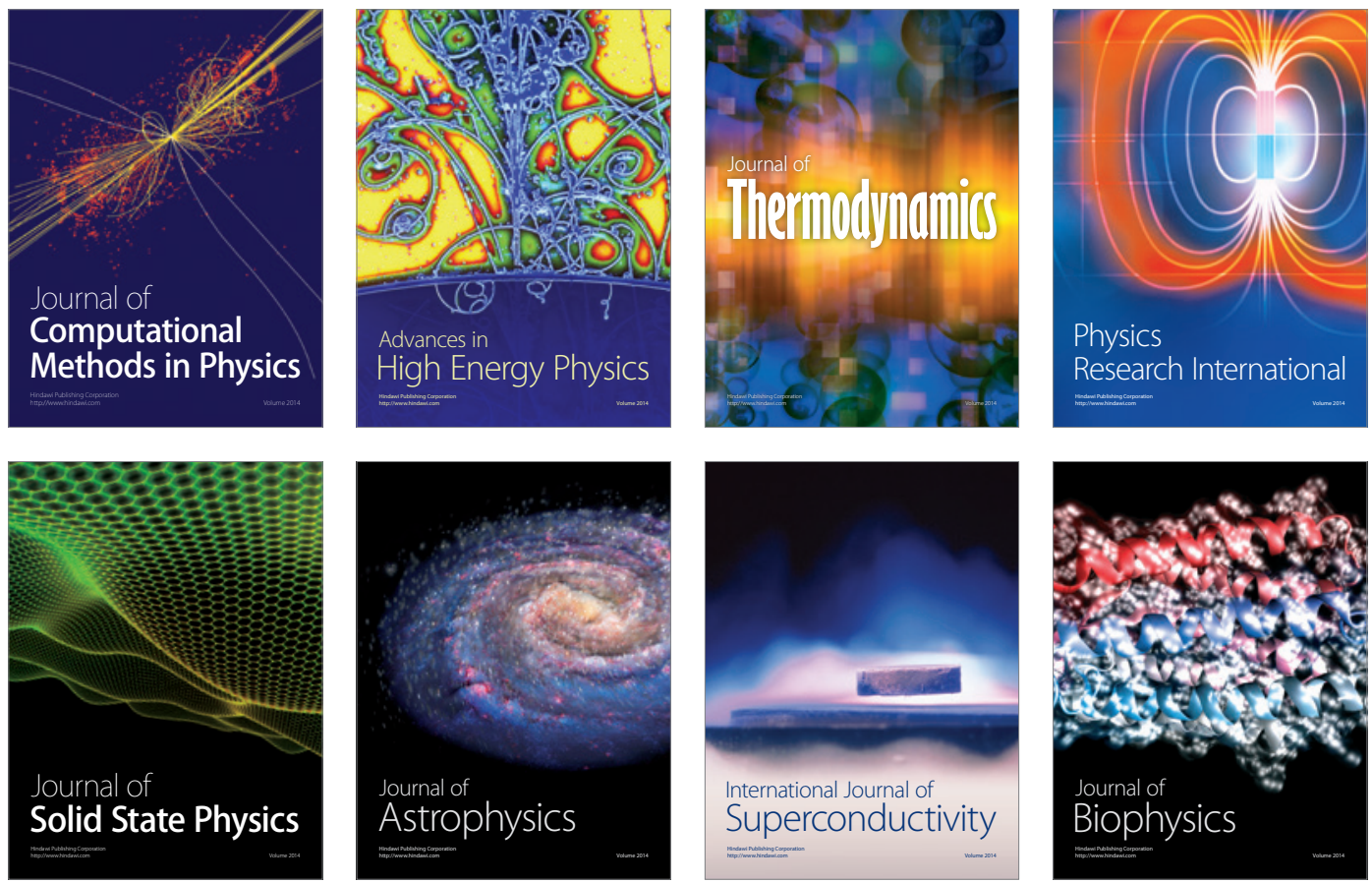
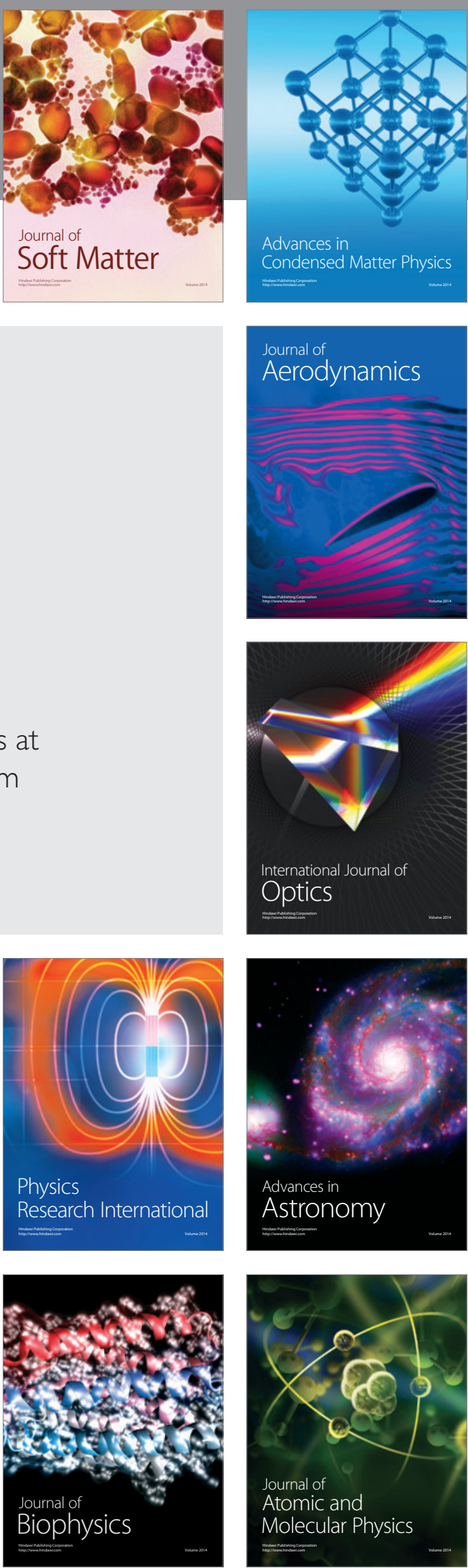